\title{
Acyl Radical Smiles Rearrangement To Construct Hydroxybenzophenones by
}

\section{Photoredox Catalysis}

\author{
Junzhao $\mathrm{Li}^{1}$, Zhengyi Liu ${ }^{1}$, Shuang $\mathrm{Wu}^{1,2}$ and Prof. Dr. Yiyun Chen ${ }^{1,2^{*}}$
}

\section{Supplementary Information}

\section{Content}

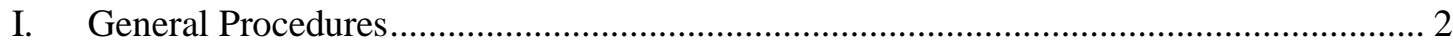

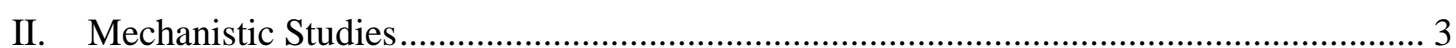

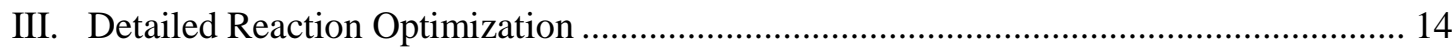

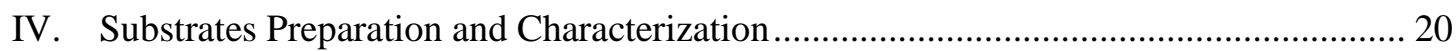

V. Smiles Rearrangement Product Characterization .............................................................. 33

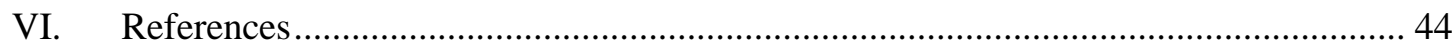




\section{General Procedures}

Unless otherwise noted, all reactions of substrates preparation were conducted in flame-dried glassware under a nitrogen atmosphere using anhydrous solvent passed through an activated alumina column (Innovative Technology). Commercially available reagents were used without further purification. Thin layer chromatography (TLC) was performed using Jiangyou TLC silica gel plates HSG $F_{254}$ and visualized using UV light, anisaldehyde or potassium permanganate. Flash chromatography was performed on Lisure science EZ purification system using the Santai technologies silica gel cartridge. Photochemical reactions were carried with $4 \mathrm{~W}$ blue LED (468 $\mathrm{nm}$ peak wavelength, $25 \mathrm{~nm}$ spectral half-wave width, composed of 55-65 LED units each with $60 \mathrm{~mW}, 3 \mathrm{~V}, 20 \mathrm{~mA}$ ) obtained from Qiding Photo Electric (analyzed by Everfine PMS-50). ${ }^{1} \mathrm{H}$ and ${ }^{13} \mathrm{C}$ NMR spectra were recorded in chloroform-d or $\left(\mathrm{CD}_{3}\right)_{2} \mathrm{SO}$, unless otherwise noted, on a Bruker AV-400 MHz or an Agilent $500 \mathrm{MHz}$ spectrometer. Chemical shifts in ${ }^{1} \mathrm{H}$ NMR spectra were reported in parts per million $(\mathrm{ppm})$ on the $\delta$ scale from an internal standard of residual chloroform $(7.26 \mathrm{ppm})$ or dimethyl sulfoxide (2.50 ppm). Data for ${ }^{1} \mathrm{H}$ NMR were reported as follows: chemical shift, multiplicity ( $\mathrm{s}=$ singlet, $\mathrm{d}=$ doublet, $\mathrm{t}=$ triplet, $\mathrm{q}=$ quartet, $\mathrm{m}=$ multiplet $)$, coupling constant in Herts $(\mathrm{Hz})$ and integration. Data for ${ }^{13} \mathrm{C}$ NMR spectra were reported in terms of the chemical shift in ppm from the central peak of chloroform-d $(77.16 \mathrm{ppm})$ or $\left(\mathrm{CD}_{3}\right)_{2} \mathrm{SO}(39.52 \mathrm{ppm})$. IR (neat) spectra were recorded on a Thermo Scientific Nicolet 380 FT-IR (neat) spectrometer. MS experiments were performed on a Bruker maXis 4G instrument for HRMS-ESI, an Agilent 5973N instrument for EI-MS, and a Waters Micromass GCT Premier instrument for HRMS-EI. Cyclic voltammetry experiments were performed on a $\mathrm{CH}$ Instruments Electrochemical Analyzer. UV-VIS absorption spectrum and the quantum yield measurement were recorded using a HITACHI U-3700 Spectrometer. 


\section{Mechanistic Studies}

\section{Characterization of the BI-Ketoacid Complex}<smiles>COc1cc(C)c(Oc2ccccc2C(=O)O)c(C)c1</smiles>

$1 \mathrm{a}$<smiles>Cc1cc(C)c(Oc2ccccc2C(=O)C(=O)OI2OC(=O)c3ccccc32)c(C)c1</smiles>

$343 \%$

Scheme S1 The synthesis of the BI-ketoacid complex

Ketoacid 1a (854 mg, $3 \mathrm{mmol})$, BIOH (1.06 g, $4 \mathrm{mmol})$ and $10 \mathrm{~mL}$ DCM were added to a $25 \mathrm{~mL}$ round-bottomed flask equipped with a stir bar and $4 \AA$ MS (1 g), and stirred at room temperature for $6 \mathrm{~h}$ in the dark. ${ }^{1}$ After filtration and concentration in vacuo, $10 \mathrm{~mL} \mathrm{Et}_{2} \mathrm{O}$ was added, and the precipitation was washed by $\mathrm{Et}_{2} \mathrm{O}(10$ mL*3). The reaction afforded 3 as a pale-yellow solid (688 mg, 43\%). ${ }^{1} \mathrm{H}$ NMR (400 $\left.\mathrm{MHz} \mathrm{CDCl}_{3}\right) \delta 8.27-8.18(\mathrm{~m}, 1 \mathrm{H}), 8.10-8.03(\mathrm{~m}, 1 \mathrm{H}), 7.97(\mathrm{~d}, J=9.3 \mathrm{~Hz}, 1 \mathrm{H})$, $7.66(\mathrm{dd}, J=6.2,2.2 \mathrm{~Hz}, 2 \mathrm{H}), 7.43(\mathrm{ddd}, J=8.5,7.5,1.8 \mathrm{~Hz}, 1 \mathrm{H}), 7.11(\mathrm{t}, J=7.6 \mathrm{~Hz}$, 1H), $6.88(\mathrm{~s}, 2 \mathrm{H}), 6.43(\mathrm{~d}, J=8.3 \mathrm{~Hz}, 1 \mathrm{H}), 2.28(\mathrm{~s}, 3 \mathrm{H}), 2.02(\mathrm{~s}, 6 \mathrm{H}) ;{ }^{13} \mathrm{C}$ NMR $(126$ $\left.\mathrm{MHz}, \mathrm{CDCl}_{3}\right) \delta 184.38,170.07,167.86,158.88,147.44,136.95,136.64,135.90$, $133.47,131.66,131.27,130.77,130.17,128.85,128.37,122.13,121.38,118.61$, 113.32, 20.91, 16.27; IR (neat): 2922, 1732, 17040, 1682, 1596, 1474, 1453, 1155,

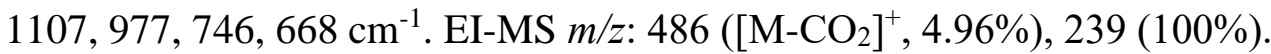

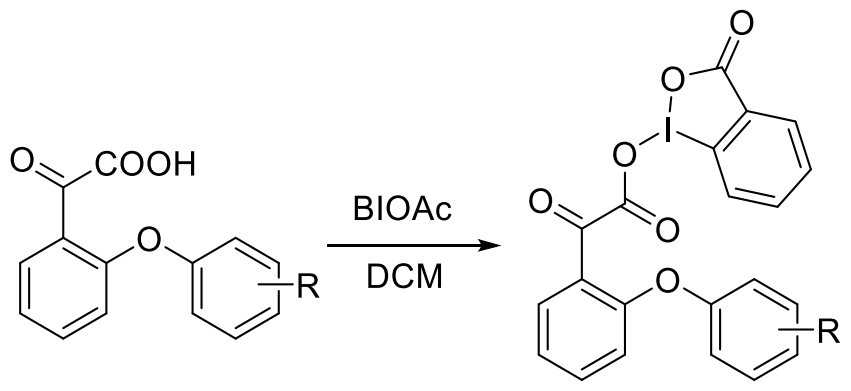

Following the literature procedure with slight modifications, the ketoacid (1.2 mmol), BI-OAc (306 mg, 1.0 eq. $1 \mathrm{mmol}$ ) ${ }^{1}, \mathrm{CH}_{2} \mathrm{Cl}_{2}$ were mixed and stirred at room 
temperature for $12 \mathrm{~h}$. After filtration and concentration, $10 \mathrm{ml} \mathrm{Et} 2 \mathrm{O}$ was added. The precipitation was washed by $\mathrm{Et}_{2} \mathrm{O}(10 \mathrm{~mL} * 3)$.<smiles>CCOC(=O)c1ccc(Oc2ccccc2C(=O)C(=O)OI2OC(=O)c3ccccc32)cc1</smiles>

Ethyl-4-(2-(2-oxo-2-((3-oxo- $\lambda^{3}$-benzo[d][1,2]iodaoxol-1(3H)-yl)oxy)acetyl)phenox y)benzoate. The reaction of 1 f afforded BI-1f complex as a pale-yellow solid (207 mg, 37\%). ${ }^{1} \mathrm{H}$ NMR (500 MHz, $\left.\mathrm{CDCl}_{3}\right) \delta 8.20(\mathrm{dd}, J=7.5,1.4 \mathrm{~Hz}, 1 \mathrm{H}), 8.09(\mathrm{dd}, J=$ 7.9, $1.7 \mathrm{~Hz}, 1 \mathrm{H}), 7.93$ (d, $J=8.6 \mathrm{~Hz}, 2 \mathrm{H}), 7.83$ (dt, $J=16.1,8.2 \mathrm{~Hz}, 3 \mathrm{H}), 7.71-7.62$ (m, 2H), 7.37 (t, $J=7.6 \mathrm{~Hz}, 1 \mathrm{H}), 7.01$ (dd, $J=8.3,6.3 \mathrm{~Hz}, 3 \mathrm{H}), 4.34$ (q, $J=7.1 \mathrm{~Hz}$, 2H), $1.37(\mathrm{t}, J=7.1 \mathrm{~Hz}, 3 \mathrm{H}) ;{ }^{13} \mathrm{C} \mathrm{NMR}\left(126 \mathrm{MHz}, \mathrm{CDCl}_{3}\right) \delta 184.07,169.23,167.74$, $165.57,159.96,156.85,136.71,136.65,133.46,132.02,131.63,131.23,129.01$, 128.06, 126.69, 125.54, 125.21, 120.04, 118.36, 118.04, 61.27, 14.43; IR (neat): 3075, $2975,1706,1682,1600,1576,1477,1453,1342,1265,1238,1161,1107,980,827$, $744,693,669,644 \mathrm{~cm}^{-1}$.<smiles>O=C(OI1OC(=O)c2ccccc21)C(=O)c1ccccc1Oc1ccc([N+](=O)[O-])cc1</smiles>

3-oxo- $\lambda^{3}$-benzo[d][1,2]iodaoxol-1(3H)-yl-2-(2-(4-nitrophenoxy)phenyl)-2-oxoacet ate The reaction of $\mathbf{1 h}$ afforded BI-1h complex as a pale-yellow solid (305 $\mathrm{mg}, 57 \%)$. ${ }^{1} \mathrm{H}$ NMR (500 MHz, $\left.\mathrm{CDCl}_{3}\right) \delta 8.23(\mathrm{dd}, J=7.6,1.2 \mathrm{~Hz}, 1 \mathrm{H}), 8.19-8.13(\mathrm{~m}, 2 \mathrm{H})$, $8.11(\mathrm{dd}, J=7.9,1.6 \mathrm{~Hz}, 1 \mathrm{H}), 7.90-7.82(\mathrm{~m}, 2 \mathrm{H}), 7.76-7.67(\mathrm{~m}, 2 \mathrm{H}), 7.44(\mathrm{t}, J=$ $7.6 \mathrm{~Hz}, 1 \mathrm{H}), 7.08$ (d, $J=9.2 \mathrm{~Hz}, 2 \mathrm{H}), 7.04$ (d, $J=8.2 \mathrm{~Hz}, 1 \mathrm{H}) .{ }^{13} \mathrm{C}$ NMR $(126 \mathrm{MHz}$, $\left.\mathrm{CDCl}_{3}\right) \delta 183.73,169.09,167.59,161.61,155.89,143.89,136.84,136.81,133.72$, 131.87, 131.64, 129.02, 128.01, 126.28, 126.09, 125.86, 120.61, 118.23, 118.17; IR 
(neat): 1702, 1682, 1598, 1571, 1516, 1477, 1343, 1264, 1238, 1203, 1109, 977, 821, $745,692,666 \mathrm{~cm}^{-1}$.<smiles>COc1cccc(OC)c1OC(=O)C(=O)OI1OC(=O)c2ccccc21</smiles>

3-oxo- $\lambda^{3}$-benzo[d][1,2]iodaoxol-1(3H)-yl-2-(2-(2,6-dimethoxyphenoxy)phenyl)-2-o xoacetate The reaction of $\mathbf{1 i}$ afforded BI-1i complex as a pale-yellow solid (268 $\mathrm{mg}$, 49\%). ${ }^{1} \mathrm{H}$ NMR (500 MHz, $\left.\mathrm{CDCl}_{3}\right) \delta 8.20(\mathrm{dd}, J=7.6,1.5 \mathrm{~Hz}, 1 \mathrm{H}), 8.00$ (dd, $J=7.8$, $1.7 \mathrm{~Hz}, 1 \mathrm{H}), 7.96$ (d, $J=8.2 \mathrm{~Hz}, 1 \mathrm{H}), 7.79$ (ddd, $J=8.5,7.3,1.6 \mathrm{~Hz}, 1 \mathrm{H}), 7.71-7.65$ (m, 1H), 7.44 (ddd, $J=8.6,7.3,1.8 \mathrm{~Hz}, 1 \mathrm{H}), 7.17-7.09(\mathrm{~m}, 2 \mathrm{H}), 6.57-6.61(\mathrm{~m}, 3 \mathrm{H})$, $3.63(\mathrm{~s}, 6 \mathrm{H}) ;{ }^{13} \mathrm{C} \mathrm{NMR}\left(126 \mathrm{MHz}, \mathrm{CDCl}_{3}\right) \delta 184.62,169.43,168.10,159.52,153.07$, $136.52,136.31,136.28,133.15,131.44,130.47,129.03,128.40,126.52,122.26$, 122.24, 118.91, 114.22, 105.71, 56.22; IR (neat): 3006, 2948, 2840, 1702, 1679, 1599, $1495,1480,1304,1260,1238,1155,1110,978,741,694,669 \mathrm{~cm}^{-1}$.

\section{Reactivity of the BI-Ketoacid Complex}

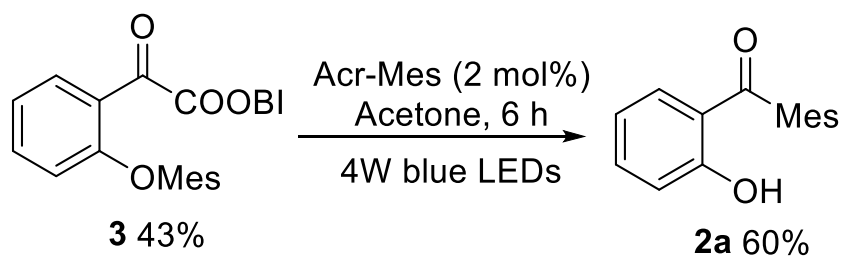

BI-ketoacid complex 3 (53.0 mg, $0.10 \mathrm{mmol})$, Acr-Mes-ClO $4(0.8 \mathrm{mg}, 0.002$ mmol, 0.02 eq.) were placed in a $10 \mathrm{~mL}$ schlenk tube equipped with a magnetic stir bar. After $2.0 \mathrm{~mL}$ Acetone (treated by $4 \AA \mathrm{MS}$ ) was added, the reaction mixture was degassed using a freeze-pump-thaw procedure (3 times) and exposed to blue LEDs (two $4 \mathrm{~W}$ LED light bulbs $10 \mathrm{~cm}$ away from the vial, $468 \mathrm{~nm}$ peak wavelength, $25 \mathrm{~nm}$ spectral half-wave width, composed of 55-65 LED units each with $60 \mathrm{~mW}, 3 \mathrm{~V}, 20$ 
$\mathrm{mA}$ ) at room temperature with stirring for $6 \mathrm{~h}$. The reaction mixture concentrated and purified directly by column chromatography to afford the product $\mathbf{1 a}(14.4 \mathrm{mg}, 60 \%)$.

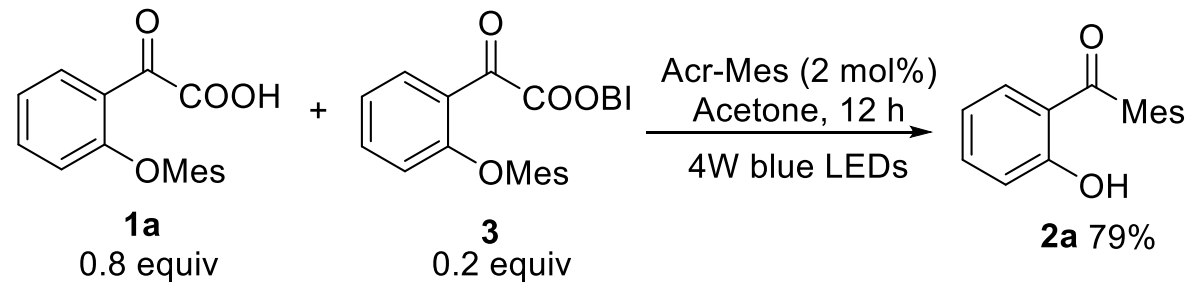

BI-ketoacid complex 3 (10.8 mg, 0.02 mmol, 0.2 eq), ketoacid 1a (22.7 mg, 0.08 mmol, 0.8 eq $)$, and Acr-Mes- $\mathrm{ClO}_{4}(0.8 \mathrm{mg}, 0.002 \mathrm{mmol}, 0.02$ eq. $)$ were placed in a 10 $\mathrm{mL}$ schlenk tube equipped with a magnetic stir bar. After $2.0 \mathrm{~mL}$ Acetone (treated by $4 \AA$ MS) was added, the reaction mixture was degassed using a freeze-pump-thaw procedure (3 times) and exposed to blue LEDs (two $4 \mathrm{~W}$ LED light bulbs $10 \mathrm{~cm}$ away from the vial, at room temperature with stirring for 1 $2 \mathrm{~h}$. The reaction mixture concentrated and purified directly by column chromatography to afford the product $\mathbf{2 a}(19.0 \mathrm{mg}, 79 \%)$. 


\section{The Radical Trapping Experiment}

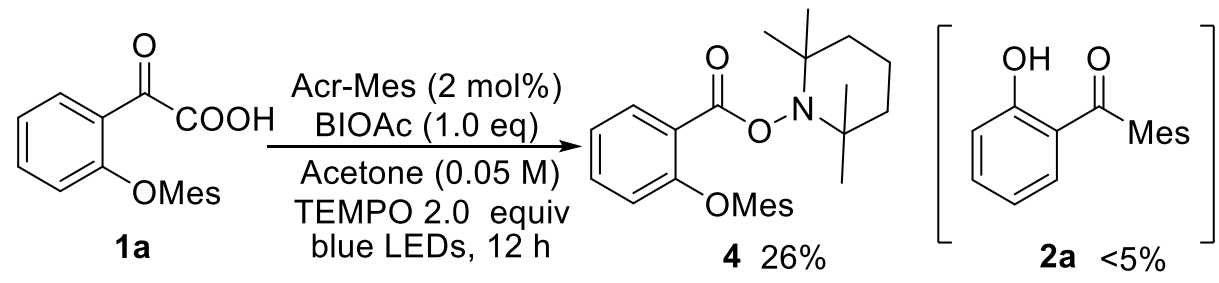

Scheme S2 The Radical Trapping Experiment ${ }^{2}$

$1 \mathrm{a}(28.4 \mathrm{mg}, 0.10 \mathrm{mmol})$, Acr-Mes- $\mathrm{ClO}_{4}(0.8 \mathrm{mg}, 0.002 \mathrm{mmol}, 0.02 \mathrm{eq}$.$) , BIOAc$ (1.0 equiv.), and TEMPO (31.2 mg, 2.0 equiv.) were placed in a $10 \mathrm{~mL}$ schlenk tube vial equipped with a magnetic stir bar. After $2.0 \mathrm{~mL}$ Acetone (treated by 4 A MS) was added into the tube via a syringe, the reaction mixture was degassed using a freeze-pump-thaw procedure ( 3 times) and exposed to blue LEDs for $12 \mathrm{~h}$. The crude product was purified by removing TEMPO in vacuo and subsequent column chromatography $\left(\mathrm{R}_{\mathrm{f}}=0.65, \quad \mathrm{PE} / \mathrm{EA}=10: 1\right)$ to afford the TEMPO-addition adduct 4 as a colorless liquid (10.5 mg, 26\%). ${ }^{1} \mathrm{H}$ NMR (500 MHz, $\left.\mathrm{CDCl}_{3}\right) \delta 7.87(\mathrm{~d}, J=7.6$ $\mathrm{Hz}, 1 \mathrm{H}), 7.30-7.24(\mathrm{~m}, 1 \mathrm{H}), 7.00(\mathrm{t}, J=7.5 \mathrm{~Hz}, 1 \mathrm{H}), 6.92(\mathrm{~s}, 2 \mathrm{H}), 6.40$ (d, J = 8.4 Hz, 1H), $2.31(\mathrm{~s}, 3 \mathrm{H}), 2.10(\mathrm{~s}, 6 \mathrm{H}), 1.79(\mathrm{t}, J=13.5 \mathrm{~Hz}, 2 \mathrm{H}), 1.67(\mathrm{t}, J=13.3 \mathrm{~Hz}, 2 \mathrm{H}), 1.57$ $(\mathrm{d}, J=12.9 \mathrm{~Hz}, 12 \mathrm{H}) ;{ }^{13} \mathrm{C} \mathrm{NMR}\left(126 \mathrm{MHz}, \mathrm{CDCl}_{3}\right) \delta 167.88,156.66,148.29,134.72$, $133.06,132.38,130.78,129.73,120.90,119.71,113.21,60.34,39.14,32.01,20.89$, 17.09, 16.23; IR (neat): 2969, 2928, 2867, 1737, 1600, 1478, 1448, 1381, 1362, 1284, 1231, 1200, 1037, 851, $752 \mathrm{~cm}^{-1}$; HRMS-ESI (m/z): $[\mathrm{M}+\mathrm{H}]^{+}$calc'd for $\mathrm{C}_{25} \mathrm{H}_{34} \mathrm{NO}_{3}$ 396.2533, found 396.2538. 


\section{The Radical Crossover Experiments}<smiles>COc1ccc(C(=O)C(=O)O)c(Oc2c(C)cc(C)cc2C)c1</smiles><smiles>CCCc1cccc(CCC)c1Oc1ccccc1C(=O)C(=O)O</smiles>

$1 \mathrm{j}$
Acr-Mes (2 mol\%) BIOAc (20 mol\%)

Acetone $(0.05 \mathrm{M})$ Blue LEDs $\mathrm{N}_{2}, 12 \mathrm{~h}$<smiles>COc1ccc(C(=O)c2c(C)cc(C)cc2C)c(O)c1</smiles>

2q $85 \%$<smiles>COc1ccc(C(=O)c2c(C(C)C)cccc2C(C)C)c(O)c1</smiles><smiles>CCCc1cccc(CCC)c1C(=O)c1ccccc1O</smiles>

2j $73 \%$<smiles>Cc1cc(C)c(C(=O)c2ccccc2O)c(C)c1</smiles>

\section{Scheme S3 The Radical Crossover Experiments}

Ketoacid 1j (0.10 mmol, 1.0 equiv.), ketoacid 1q (0.10 mmol, 1.0 equiv.), Acr-Mes ${ }^{+} \mathrm{ClO}_{4}{ }^{-}(1.6 \mathrm{mg}, 0.004 \mathrm{mmol}, 0.02$ equiv.), and BIOAc (12.2 mg, $0.04 \mathrm{mmol}$, 0.2 equiv.) were placed in a $10 \mathrm{~mL}$ schlenk tube vial equipped with a magnetic stir bar. After $4.0 \mathrm{~mL}$ Acetone (treated by $4 \AA \mathrm{MS}$ ) was added into the tube via a syringe, the reaction mixture was degassed using a freeze-pump-thaw procedure ( 3 times) and exposed to blue LEDs at room temperature. After stirring for $12 \mathrm{~h}$, the reaction mixture was concentrated and purified directly by column chromatography to afford the products $\mathbf{2 j}$ ( $20.5 \mathrm{mg}, 73 \%)$ and $\mathbf{2 q}(23.0 \mathrm{mg}, 85 \%)$. 


\section{The Cyclic Voltammetry Experiment}

Cyclic voltammetry experiments were performed on a $\mathrm{CH}$ Instruments Electrochemical Analyzer at room temperature under a nitrogen atmosphere. The 0.5 $\mathrm{mM} \mathrm{CH}_{3} \mathrm{CN}$ solution of the sample was prepared with $100 \mathrm{mM}$ tetrabutylammonium hexafluorophosphate as the supporting electrolyte, using a glassy carbon working electrode, a Pt counter electrode, and SCE reference electrode. Scan Rate (V/s) = 0.1. We quantified the potential against the standard of $\mathrm{Fc} / \mathrm{Fc}^{+}$.
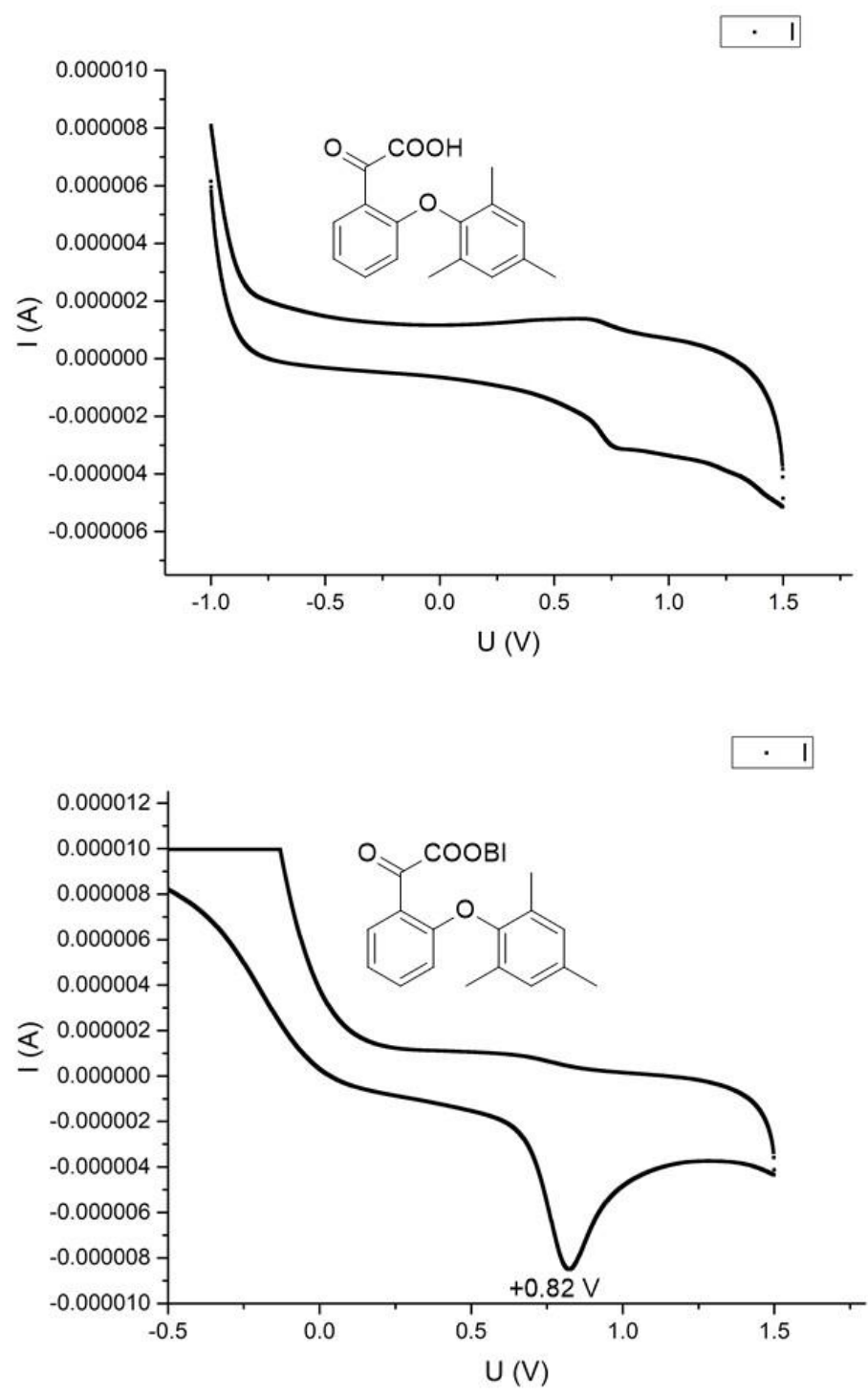

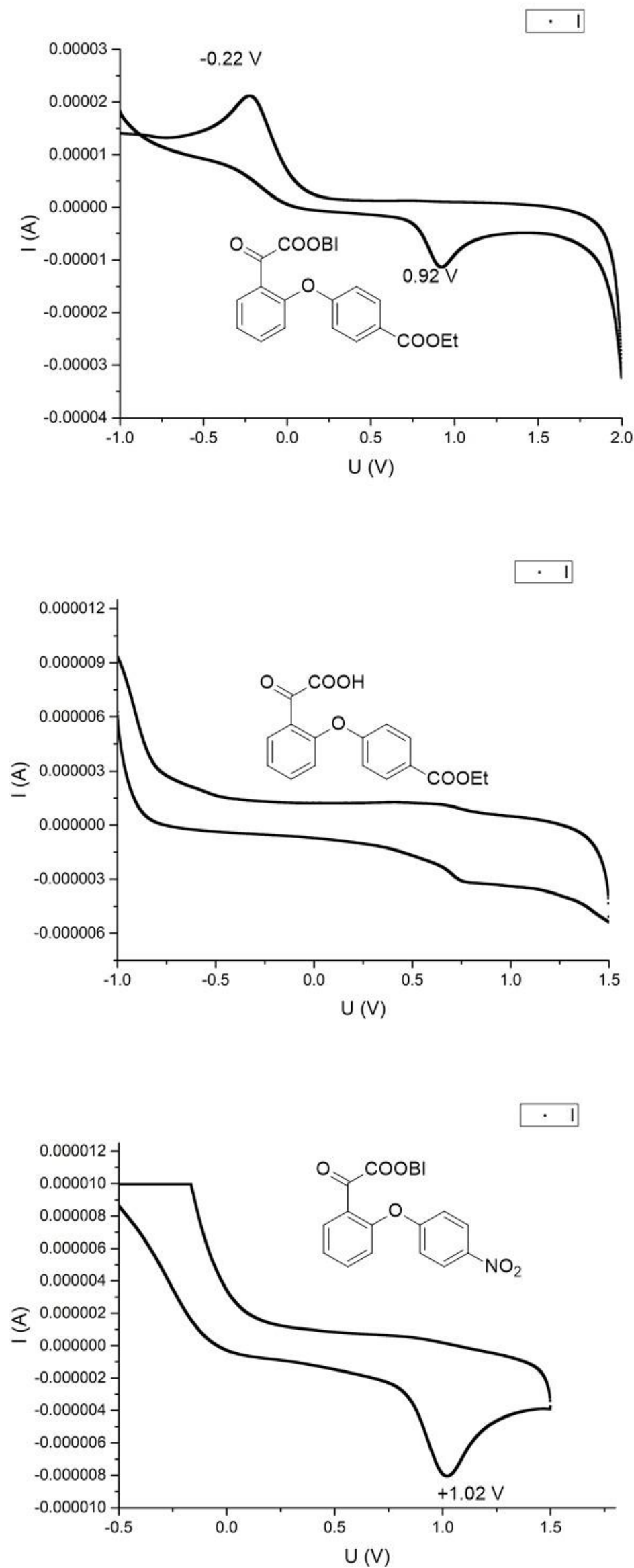

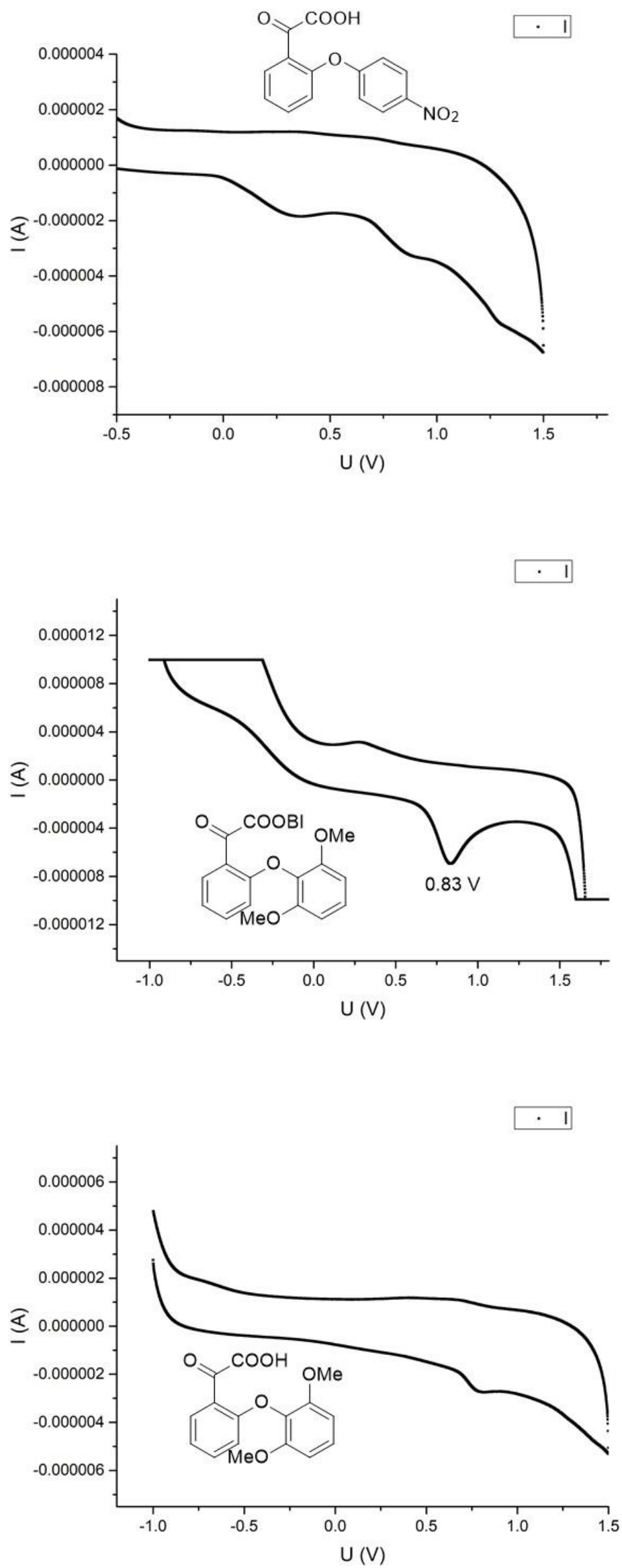


\section{Quantum Yield Measurement}

\section{Determination of the light intensity at $468 \mathrm{~nm}$}

According to the literature procedure ${ }^{3}$ the photon flux of the spectrophotometer was determined by the standard ferrioxalate actinometry. A $0.15 \mathrm{M}$ solution of ferrioxalate was prepared by dissolving potassium ferrioxalate hydrate (1.842 g) in $\mathrm{H}_{2} \mathrm{SO}_{4}(25 \mathrm{~mL}$ of a $0.05 \mathrm{M}$ solution); A buffered solution of and sodium acetate (15.75 $\mathrm{g}$ ) in $\mathrm{H}_{2} \mathrm{SO}_{4}$ (70 $\mathrm{mL}$ of a $0.5 \mathrm{M}$ solution); 1,10-phenanthroline was prepared by dissolving phenanthroline $(50 \mathrm{mg})$ in the buffer solution $(50 \mathrm{~mL})$ Both solutions were stored in the dark.

To determine the photon flux of the spectrophotometer, the ferrioxalate solution $(2.0$ $\mathrm{mL}$ ) was placed in a cuvette and irradiated for 90 seconds at $\lambda=468 \mathrm{~nm}$ ( $4 \mathrm{~W}$ blue LED). After irradiation, all the actinometer solution was placed in an $8 \mathrm{~mL}$ brown bottle. The phenanthroline solution $(0.35 \mathrm{~mL})$ and a buffer solution $(2.65 \mathrm{~mL})$ were added to the cuvette, and the mixture was allowed to stir in the dark for 1 hour to allow the ferrous ions to completely coordinate to the phenanthroline. The absorbance of the solution was measured at $510 \mathrm{~nm}\left(\mathrm{~A}_{\text {meas }}\right)$. A non-irradiated sample was also prepared and the absorbance at $510 \mathrm{~nm}$ was measured $\left(\mathrm{A}_{\mathrm{ref}}\right)$. The conversion was calculated using equation (1). Where $\mathrm{V}$ is the total volume $(5 \mathrm{~mL})$ of the solution after addition of phenanthroline, 1 is the path length $(1.000 \mathrm{~cm})$, and $\varepsilon$ is the molar absorptivity at $510 \mathrm{~nm}\left(11,100 \mathrm{~L} \mathrm{~mol}^{-1} \mathrm{~cm}^{-1}\right)$.

$$
\begin{gathered}
\mathrm{mol} \mathrm{Fe} e^{2+}=\frac{V\left(A_{\text {meas }}-A_{\text {ref }}\right)}{\varepsilon b} \\
m o l ~ F e^{2+}=\frac{5 m L *(1.3406-0.2556)}{11100 \mathrm{Lol}^{-1} \mathrm{~cm}^{-1} \times 1.000 \mathrm{~cm}}=4.887 \times 10^{-7} \mathrm{~mol}
\end{gathered}
$$

The photon flux was calculated using equation (2) where $\Phi$ is the quantum yield for the ferrioxalate actinometer $\left(0.92\right.$ for a $0.15 \mathrm{M}$ solution at $\left.\lambda=468 \mathrm{~nm}^{4}\right), \mathrm{t}$ is the time (90.0 s), and $\mathrm{f}$ is the fraction of light absorbed at $\lambda=468 \mathrm{~nm}$ (equation (3)). The absorbance of the above ferrioxalate solution at $468 \mathrm{~nm}$ was measured to be 0.5186 , indicating the fraction of light absorbed (f) is 0.6970 .

$$
\text { Photon flux }=\frac{\mathrm{mol} \mathrm{Fe}^{2+}}{\Phi \cdot t \cdot f}=\frac{4.887 \times 10^{-7} \mathrm{~mol}}{0.92 \times 90 \times 0.6970}=8.469 \times 10^{-9} \text { einstein }^{-1}
$$




$$
\mathrm{f}=1-10^{-0.5186}=0.6970
$$

\section{Determination of quantum yield:}

A cuvette was charged with $1 \mathrm{a}(0.1 \mathrm{mmol}, 1$ equiv), BIOAc (0.02 mmol, $20 \mathrm{~mol} \%)$, Acr-Mes (0.002 mmol, $2 \mathrm{~mol} \%)$, and $2.0 \mathrm{~mL}$ Acetone. The cuvette was then degassed with $\mathrm{N}_{2}$, then capped with a PTFE stopper. The sample was stirred and irradiated (4W blue LED, $\lambda=468 \mathrm{~nm})$ for $5400 \mathrm{~s}(1.5 \mathrm{~h})$. After irradiation, the yield of product formed was determined by ${ }^{1} \mathrm{H}$ NMR to be $16 \%$ using p-nitrobenzyl alcohol as an external standard. The quantum yield was determined using equation (4). The absorbance of Acr-Mes in Acetone was measured at the reaction concentration of $1 \times$ $10^{-3} \mathrm{M}$. The absorbance at $468 \mathrm{~nm}$ is 0.5194 indicating the fraction of light absorbed is 0.6976 .

$$
\begin{gathered}
\Phi=\frac{\text { mol product }}{\text { flux } \cdot t \cdot f}=\frac{1.6 \times 10^{-5} \mathrm{~mol}}{8.469 \times 10^{-9} \text { einstein } \mathrm{s}^{-1} \times 5400 \mathrm{~s} \times 0.6976}=50 \% \\
\mathrm{f}=1-10^{-0.5194}=0.6976
\end{gathered}
$$




\section{Detailed Reaction Optimization}

Table S1 The screening of BIOAc equivalence and solvents

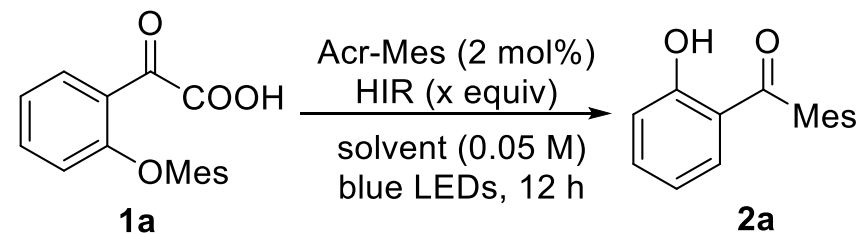

\begin{tabular}{|c|c|c|c|c|c|}
\hline Entry & HIR & solvent & Conv. & NMR Yield & Note \\
\hline 1 & / & Acetone & $5 \%$ & $6 \%$ & \\
\hline 2 & $0.1 \mathrm{eq} \mathrm{BIOAc}$ & Acetone & $83 \%$ & $73 \%$ & \\
\hline 3 & $0.2 \mathrm{eq} \mathrm{BIOAc}$ & Acetone & $93 \%$ & $87 \%(84 \%)$ & \\
\hline 4 & $0.3 \mathrm{eq}$ BIOAc & Acetone & $>95 \%$ & $85 \%(80 \%)$ & \\
\hline 5 & $0.6 \mathrm{eq}$ BIOAc & Acetone & $>95 \%$ & $77 \%(76 \%)$ & \\
\hline 6 & $1.5 \mathrm{eq}$ BIOAc & Acetone & $>95 \%$ & $58 \%(55 \%)$ & $6 \mathrm{~h}$ \\
\hline 7 & $0.2 \mathrm{eq} \mathrm{BIOAc}$ & $\mathrm{CHCl}_{3}$ & $51 \%$ & $33 \%$ & \\
\hline 8 & $0.2 \mathrm{eq} \mathrm{BIOAc}$ & $\mathrm{CH}_{3} \mathrm{CN}$ & $90 \%$ & $74 \%$ & \\
\hline 9 & $0.2 \mathrm{eq} \mathrm{BIOAc}$ & toluene & $68 \%$ & $62 \%$ & \\
\hline 10 & 0.2 eq PIDA & Acetone & $>95 \%$ & $74 \%$ & \\
\hline 11 & 0.2 eq PIFA & Acetone & $<5 \%$ & $<5 \%$ & \\
\hline 12 & $0.2 \mathrm{eq} \mathrm{BIOAc}$ & Acetone & / & / & dark \\
\hline 13 & 0.2 eq BIOAc & Acetone & I & l & No Acr-Mes ${ }^{+}$ \\
\hline
\end{tabular}

${ }^{a}$ Reaction conditions: 1 (0.1 mmol, 1.0 equiv.), Acr-Mes ${ }^{+} \mathrm{ClO}_{4}{ }^{-}$

(0.002 mmol, $2 \mathrm{~mol} \%$ ), and BIOAc in $2 \mathrm{~mL}$ solvent with $4 \mathrm{~W}$ blue

LEDs irradiation at room temperature for $12 \mathrm{~h} .{ }^{b}$ Conversions and

Yields were determined by ${ }^{1} \mathrm{H}-\mathrm{NMR}$ analysis. Yields of isolated

products are given in parentheses 
Table S2 The screening of additives

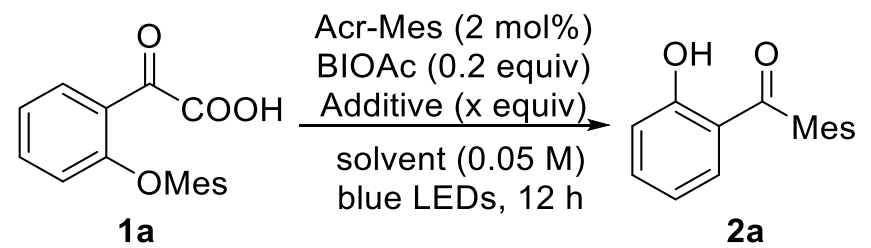

\begin{tabular}{cccccc}
\hline Entry & HIR & Additive & Conv. & Yield & $\mathrm{t} / \mathrm{h}$ \\
\hline 1 & BIOAc & 0.2 eq $\mathrm{Na}_{2} \mathrm{CO}_{3}$ & $>95 \%$ & $92 \%(83 \%)$ & $8 \mathrm{~h}$ \\
2 & BIOAc & 0.2 eq DIPEA & $>95 \%$ & $83 \%$ & $8 \mathrm{~h}$ \\
3 & BIOAc & $0.4 \mathrm{eq} \mathrm{AcOH}$ & $>95 \%$ & $82 \%$ & $12 \mathrm{~h}$ \\
4 & BIOAc & $0.4 \mathrm{eq} \mathrm{BzOH}$ & $>95 \%$ & $88 \%(82 \%)$ & $12 \mathrm{~h}$ \\
5 & BIOAc & $0.4 \mathrm{eq} \mathrm{TFA}$ & $26 \%$ & $22 \%$ & $12 \mathrm{~h}$ \\
6 & PIDA & 0.4 eq TFA & $10 \%$ & $9 \%$ & $12 \mathrm{~h}$ \\
\hline
\end{tabular}

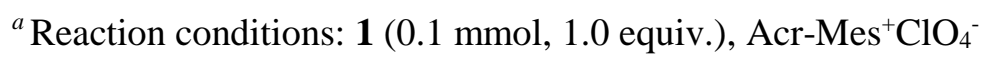

(0.002 mmol, $2 \mathrm{~mol} \%)$, HIR, and the additive in $2 \mathrm{~mL}$ solvent with $4 \mathrm{~W}$ blue LEDs irradiation at room temperature for $12 \mathrm{~h} .{ }^{b}$ Conversions and Yields were determined by ${ }^{1} \mathrm{H}-\mathrm{NMR}$ analysis. Yields of isolated products are given in parentheses 
Table S3 The screening of photocatalysts

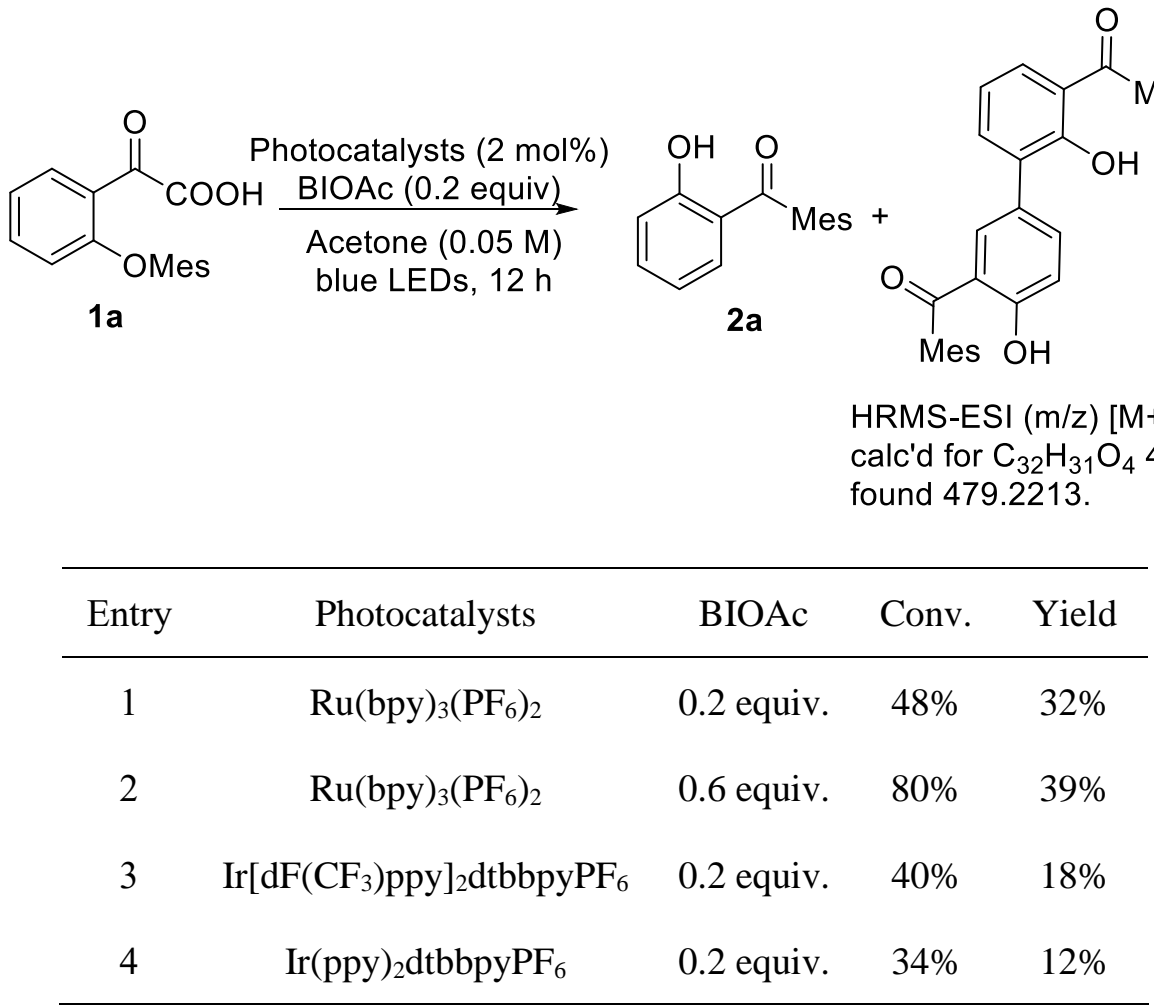

${ }^{a}$ Reaction conditions: 1 ( 0.1 mmol, 1.0 equiv.), Photocatalysts (0.002 mmol, $2 \mathrm{~mol} \%$ ), and BIOAc in $2 \mathrm{~mL}$ solvent with $4 \mathrm{~W}$ blue LEDs irradiation at room temperature for $12 \mathrm{~h} .{ }^{b}$ Conversions and Yields were determined by ${ }^{1} \mathrm{H}-\mathrm{NMR}$ analysis. Yields of isolated products are given in parentheses 
Table S4 The screening of bases

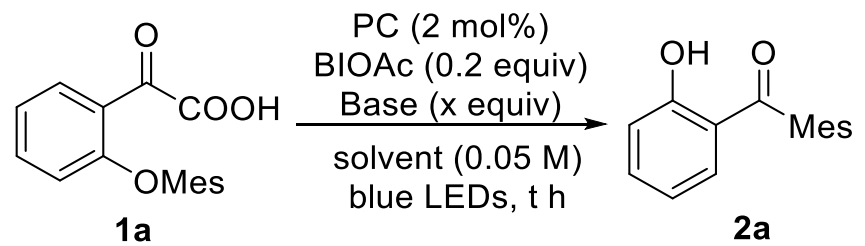

\begin{tabular}{ccccccc}
\hline Entry & Photocatalysts & Base & solvent & t/h & Conv. Yield \\
\hline 1 & Acr-Mes ${ }^{+} \mathrm{ClO}_{4}^{-}$ & $0.5 \mathrm{eq} \mathrm{K}_{2} \mathrm{HPO}_{4}$ & Acetone & 12 & $48 \%$ & $29 \%$ \\
2 & Acr-Mes $^{+} \mathrm{ClO}_{4}^{-}$ & $0.5 \mathrm{eq} \mathrm{Cs}_{2} \mathrm{CO}_{3}$ & Acetone & 12 & $37 \%$ & $20 \%$ \\
3 & Acr-Mes $^{+} \mathrm{ClO}_{4}^{-}$ & $0.5 \mathrm{eq} \mathrm{Na}_{2} \mathrm{CO}_{3}$ & Acetone & 12 & $37 \%$ & $23 \%$ \\
4 & Acr-Mes $^{+} \mathrm{ClO}_{4}^{-}$ & 0.2 eq Na $\mathrm{CO}_{3}$ & Acetone & 12 & $75 \%$ & $33 \%$ \\
5 & $\mathrm{Acr-Mes}{ }^{+} \mathrm{ClO}_{4}^{-}$ & 0.1 eq $\mathrm{K}_{2} \mathrm{HPO}_{4}$ & $\mathrm{CH}_{3} \mathrm{CN}$ & 40 & $32 \%$ & $31 \%$ \\
6 & $\mathrm{Ru}(\mathrm{bpy})_{3}\left(\mathrm{PF}_{6}\right)_{2}$ & $0.2 \mathrm{eq} \mathrm{Cs}_{2} \mathrm{CO}_{3}$ & Acetone & 14 & $<5 \%$ & $<5 \%$ \\
\hline
\end{tabular}

${ }^{a}$ Reaction conditions: 1 (0.1 mmol, 1.0 equiv.), Photocatalysts (0.002 mmol, 2mol\%), BIOAc (0.02 mmol, 0.2 equiv.), and the base in $2 \mathrm{~mL}$ solvent with $4 \mathrm{~W}$ blue LEDs irradiation at room temperature for $12 \mathrm{~h} .{ }^{b}$ Conversions and Yields were determined by ${ }^{1} \mathrm{H}-\mathrm{NMR}$ analysis. Yields of isolated products are given in parentheses 
Table S5 The aqueous reactions ${ }^{a}$

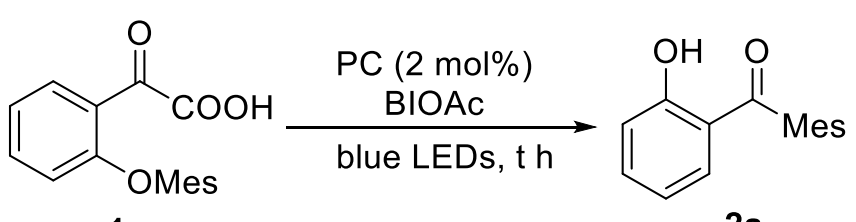

1a

$2 a$

\begin{tabular}{|c|c|c|c|c|c|}
\hline Photocatalyst & BIOAc & solvent & Conv. $^{b}$ & yield $^{b}$ & $\mathrm{t} / \mathrm{h}$ \\
\hline $2 \mathrm{~mol} \% \mathrm{Ru}(\mathrm{bpy})_{3}\left(\mathrm{PF}_{6}\right)_{2}$ & $1.0 \mathrm{eq}$ & $\mathrm{CH}_{3} \mathrm{CN}: \mathrm{PBS}^{c}=9: 110 \mathrm{mM}$ & $89 \%$ & $<28 \%$ & $3 \mathrm{~h}$ \\
\hline $2 \mathrm{~mol} \% \mathrm{Ru}(\mathrm{bpy})_{3}\left(\mathrm{PF}_{6}\right)_{2}$ & $1.0 \mathrm{eq}$ & Acetone $: \mathrm{PBS}^{c}=9: 110 \mathrm{mM}$ & $78 \%$ & $19 \%$ & $3 \mathrm{~h}$ \\
\hline $10 \mathrm{~mol} \%$ Acr-Mes ${ }^{+} \mathrm{ClO}_{4}^{-}$ & $1.0 \mathrm{eq}$ & $\mathrm{CH}_{3} \mathrm{CN}: \mathrm{PBS}^{c}=9: 110 \mathrm{mM}$ & $>95 \%$ & $66 \%$ & $6 \mathrm{~h}$ \\
\hline $10 \mathrm{~mol} \%$ Acr-Mes ${ }^{+} \mathrm{ClO}_{4}^{-}$ & $1.0 \mathrm{eq}$ & Acetone $: \mathrm{PBS}^{c}=9: 110 \mathrm{mM}$ & $>95 \%$ & $66 \%$ & $6 \mathrm{~h}$ \\
\hline $10 \mathrm{~mol} \% \mathrm{Acr}-\mathrm{Mes}^{+} \mathrm{ClO}_{4}^{-}$ & $0.4 \mathrm{eq}$ & Acetone: $\mathrm{PBS}^{c}=9: 110 \mathrm{mM}$ & $88 \%$ & $66 \%$ & $12 \mathrm{~h}$ \\
\hline
\end{tabular}

${ }^{a}$ Reaction conditions: 1 ( 0.1 mmol, 1.0 equiv) with $4 \mathrm{~W}$ blue LEDs irradiation at room temperature. ${ }^{b}$ Conversions and Yields were determined by ${ }^{1} \mathrm{H}-\mathrm{NMR}$ analysis. ${ }^{c} \mathrm{pH}=7.4$. 
The detection of BI-ketoacid complex in aqueous conditions

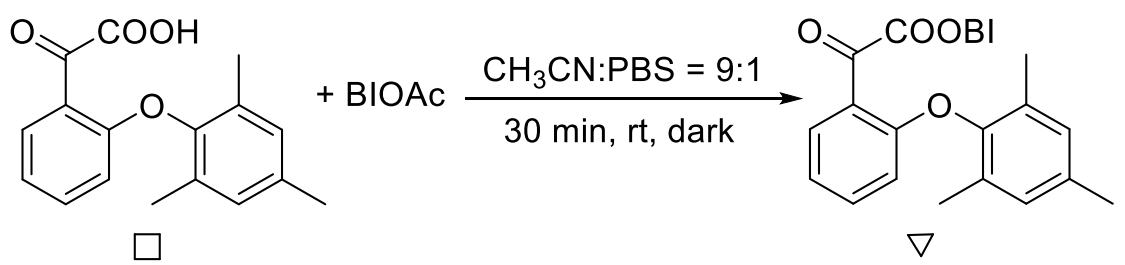

The ketoacid 1a (28.4 mg, $0.1 \mathrm{mmol})$, BI-OAc (30.6 mg, 1.0 eq. $0.1 \mathrm{mmol})$, $\mathrm{CH}_{3} \mathrm{CN}(1.8 \mathrm{~mL}), \mathrm{pH}=7.4 \mathrm{PBS}(0.2 \mathrm{~mL})$ were mixed and stirred in room temperature for $30 \mathrm{~min}$. After concentration, the residue was dissolved in $1 \mathrm{~mL}$ of $\mathrm{CDCl}_{3}$ for ${ }^{1} \mathrm{H}$ NMR analysis. The result indicated that BI-ketoacid complex formed in aqueous conditions.
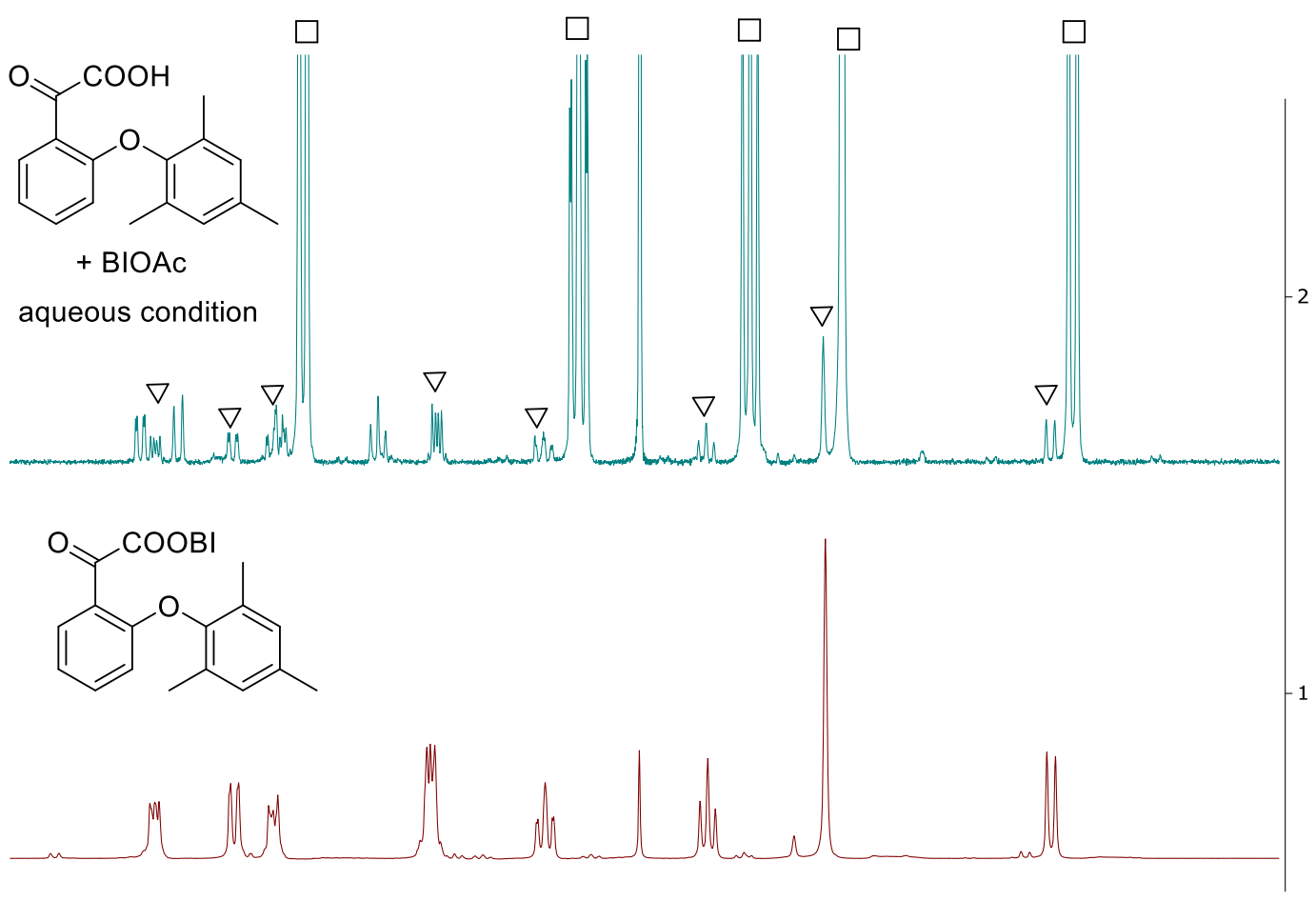

$\begin{array}{lllllllllllllllllllllllllllllllll}.5 & 8.4 & 8.3 & 8.2 & 8.1 & 8.0 & 7.9 & 7.8 & 7.7 & 7.6 & 7.5 & 7.4 & \begin{array}{l}7.3 \\ f 1(\mathrm{pm})\end{array} & 7.1 & 7.0 & 6.9 & 6.8 & 6.7 & 6.6 & 6.5 & 6.4 & 6.3 & 6.2 & 6.1\end{array}$ 


\section{Substrates Preparation and Characterization}

\section{Synthesis of ketoacid}

\section{General Procedure A ${ }^{5}{ }^{6}$}<smiles>[R]c1cccc(Oc2cc(C(C)=O)cc([R])c2C(C)=O)c1Oc1ccccc1</smiles><smiles>Cc1cc(C)c(Oc2ccccc2C(=O)O)c(C)c1</smiles>

2-(2-(mesityloxy)phenyl)-2-oxoacetic acid 1a, The general procedure A was given with 1a as a representative example. The 4-methylphenol (1.63 g, $12 \mathrm{mmol}, 1.2$ equiv.) and anhydrous $\mathrm{K}_{2} \mathrm{CO}_{3}$ (1.66 g, $12 \mathrm{mmol}$, 1.2 equiv.) were added to a solution of 2-fluorobenzketone (1.38 g, $10 \mathrm{mmol}, 1.0$ equiv) in DMA (20 mL, $0.5 \mathrm{M})$, and the resulting mixture was slowly heated to $170{ }^{\circ} \mathrm{C}$. After stirring at that temperature for 4 $\mathrm{h}$, the reaction mixture was cooled to room temperature and poured into $\mathrm{H}_{2} \mathrm{O}(40 \mathrm{~mL})$. The aqueous layer was extracted with DCM $\left(30 \mathrm{~mL}^{*} 3\right)$, dried over $\mathrm{Na}_{2} \mathrm{SO}_{4}$ with filtration, and concentrated in vacuo. The desired 2-phenoxybenzketone was obtained as a yellow solid (2.13 g, 84\%) after flash chromatography (PE / EA=50:1).

Afterwards, in a dry, single-neck, round-bottom flask equipped with a stir bar, the substituted 2-phenoxybenzketone ( $2.03 \mathrm{~g}, 8 \mathrm{mmol}, 1.0$ equiv) and selenium dioxide $\left(\mathrm{SeO}_{2}, 1.33 \mathrm{~g}, 1.5\right.$ equiv.) were added, followed by anhydrous pyridine addition (32 $\mathrm{mL}, 0.25 \mathrm{M})$. The reaction mixture was heated in an oil bath to $110{ }^{\circ} \mathrm{C}$ for $2 \mathrm{~h}$, then the bath temperature was reduced to $90{ }^{\circ} \mathrm{C}$. The mixture was stirred at this temperature $\left(90^{\circ} \mathrm{C}\right)$ for an additional $3 \mathrm{~h}$. Then most of the pyridine was removed in vacuo. The residue was added $1 \mathrm{M} \mathrm{HCl}(30 \mathrm{~mL})$ and $\mathrm{EA}\left(30 \mathrm{~mL}^{*} 2\right)$ to completely dissolve the ketoacid. The organic layer was washed with $1 \mathrm{M} \mathrm{HCl}(20 \mathrm{~mL})$, dried 
over $\mathrm{Na}_{2} \mathrm{SO}_{4}$ with filtration, and concentrated in vacuo. 1a was obtained as pale yellow solid (1.61 g, 60\%, 2 steps) after recrystallization in PE / EA = 30:1. ${ }^{1} \mathrm{H}$ NMR $\left(500 \mathrm{MHz}, \mathrm{CDCl}_{3}\right) \delta 9.73(\mathrm{~s}, 1 \mathrm{H}), 7.96(\mathrm{dd}, J=7.8,1.7 \mathrm{~Hz}, 1 \mathrm{H}), 7.45-7.36(\mathrm{~m}, 1 \mathrm{H})$, 7.07 (t, $J=7.1 \mathrm{~Hz}, 1 \mathrm{H}), 6.88(\mathrm{~s}, 2 \mathrm{H}), 6.43$ (d, $J=8.4 \mathrm{~Hz}, 1 \mathrm{H}), 2.32(\mathrm{~s}, 3 \mathrm{H}), 2.01$ (s, 6H); ${ }^{13} \mathrm{C}$ NMR $\left(126 \mathrm{MHz}, \mathrm{CDCl}_{3}\right) \delta 185.41,168.45,158.90,147.49,136.70,135.51$, 131.33, 130.86, 129.97, 121.84, 121.49, 113.20, 20.95, 16.13; IR (neat): 3039, 2922, 2611, 2561, 1720, 1673, 1597, 1474, 1458, 1267, 1196, 1153, 1139, 982, 742, 715 $\mathrm{cm}^{-1}$; HRMS-ESI $(\mathrm{m} / \mathrm{z})$ : $[\mathrm{M}-\mathrm{H}+2 \mathrm{Na}]^{+}$calc'd for $\mathrm{C}_{17} \mathrm{H}_{15} \mathrm{Na}_{2} \mathrm{O}_{4}$ 329.0760, found 329.0763 .

\section{General Procedure B ${ }^{7}$}
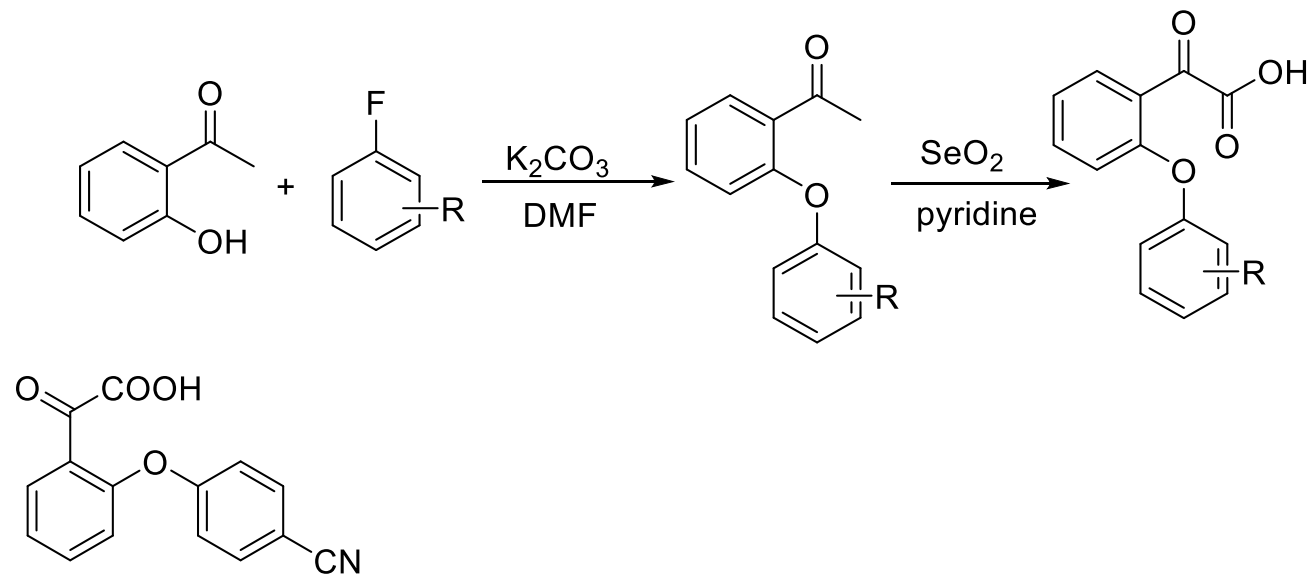

2-(2-(4-cyanophenoxy)phenyl)-2-oxoacetic acid 1e The general procedure B was given with 1e as a representative example. The phenol (1.66 g, $12 \mathrm{mmol}, 1.2$ equiv.) and anhydrous $\mathrm{K}_{2} \mathrm{CO}_{3}$ (1.66 g, $12 \mathrm{mmol}, 1.2$ equiv.) were added to a solution of fluorobenzene (1.21 g, $10 \mathrm{mmol}, 1.0$ equiv.) in DMA (20 mL, $0.5 \mathrm{M})$ and the resulting mixture was slowly heated to $100{ }^{\circ} \mathrm{C}$. After stirring at that temperature and monitored by TLC $(3 \mathrm{~h})$, the reaction mixture was cooled to room temperature and poured into $\mathrm{H}_{2} \mathrm{O}(40 \mathrm{~mL})$. The aqueous layer was extracted with DCM (30 mL*3), dried over $\mathrm{Na}_{2} \mathrm{SO}_{4}$ with filtration, and concentrated in vacuo. The desired 2-phenoxybenzketone was obtained as a pale yellow solid (1.42 g, 60\%) after flash column chromatography (PE:EA=20:1 10:1). The subsequent selenium dioxide oxidation follows the same procedure as the general procedure A to obtain 1e as a pale yellow solid (0.96 g, 36\%, 2 steps) after recrystallization in PE / EA = 10:1. ${ }^{1} \mathrm{H}$ 
$\operatorname{NMR}\left(500 \mathrm{MHz}, \mathrm{CDCl}_{3}\right) \delta 8.01(\mathrm{dd}, J=7.8,1.7 \mathrm{~Hz}, 1 \mathrm{H}), 7.66-7.61(\mathrm{~m}, 3 \mathrm{H}), 7.36(\mathrm{t}, J$ $=7.6 \mathrm{~Hz}, 1 \mathrm{H}), 7.20-6.75(\mathrm{br}, 1 \mathrm{H}), 7.10(\mathrm{~d}, J=8.9 \mathrm{~Hz}, 2 \mathrm{H}), 7.01(\mathrm{~d}, J=8.3 \mathrm{~Hz}, 1 \mathrm{H})$; ${ }^{13} \mathrm{C} \mathrm{NMR}\left(126 \mathrm{MHz}, \mathrm{CDCl}_{3}\right) \delta 185.29,166.13,159.67,155.97,136.42,134.50,131.61$, 125.64, 125.49, 119.90, 119.08, 118.37, 107.60; IR (neat): 3503, 3100, 3076, 2230, 1739, 1684, 1602, 1582, 1502, 1478, 1456, 1242, 1167, 1106, 980, 876, 840, 768, 547 $\mathrm{cm}^{-1}$; HRMS-ESI (m/z): [M-H+2Na ${ }^{+}$calc'd for $\mathrm{C}_{15} \mathrm{H}_{8} \mathrm{NNa}_{2} \mathrm{O}_{4}$ 312.0243, found 312.0248 .<smiles>O=C(O)C(=O)c1ccccc1Oc1ccccc1F</smiles>

2-(2-(2-fluorophenoxy)phenyl)-2-oxoacetic acid 1b According to procedure A, 1b was obtained as a pale yellow solid (1.58 g, 54\%, 2 steps) after recrystallization in PE / $\mathrm{DCM}=15: 1 ;{ }^{1} \mathrm{H}$ NMR $\left(500 \mathrm{MHz}, \mathrm{CDCl}_{3}\right) \delta 8.51$ (br, 1H), 7.93 (dd, $J=7.8,1.7 \mathrm{~Hz}$, 1H), 7.52 (ddd, $J=8.6,7.5,1.7 \mathrm{~Hz}, 1 \mathrm{H}), 7.24-7.05(\mathrm{~m}, 5 \mathrm{H}), 6.78(\mathrm{~d}, J=8.4 \mathrm{~Hz}, 1 \mathrm{H})$. ${ }^{13} \mathrm{C}$ NMR $\left(126 \mathrm{MHz}, \mathrm{CDCl}_{3}\right) \delta 185.49,167.45,158.17,154.33\left(\mathrm{~d},{ }^{1} J_{\mathrm{C}-\mathrm{F}}=249.4 \mathrm{~Hz}\right)$, $141.86\left(\mathrm{~d},{ }^{2} J_{\mathrm{C}-\mathrm{F}}=11.7 \mathrm{~Hz}\right), 136.29,131.14,126.40\left(\mathrm{~d},{ }^{3} J_{\mathrm{C}-\mathrm{F}}=7.0 \mathrm{~Hz}\right), 125.17\left(\mathrm{~d},{ }^{3} J_{\mathrm{C}-\mathrm{F}}\right.$ $=3.9 \mathrm{~Hz}), 123.58,123.57,122.37,117.47\left({ }^{2} J_{\mathrm{C}-\mathrm{F}}=17.9 \mathrm{~Hz}\right), 116.21 .{ }^{19} \mathrm{~F} \mathrm{NMR}(376$ $\left.\mathrm{MHz}, \mathrm{CDCl}_{3}\right) \delta-130.26$ (m); IR (neat): 3078, 1727, 1681, 1599, 1456, 1266, 1212 , 1158, 1109, $750 \mathrm{~cm}^{-1}$; HRMS-ESI (m/z): $[\mathrm{M}-\mathrm{H}+2 \mathrm{Na}]^{+}$calc'd for $\mathrm{C}_{14} \mathrm{H}_{8} \mathrm{FNa}_{2} \mathrm{O}_{4}$ 305.0197, found 305.0199.<smiles>O=C(O)C(=O)c1ccccc1Oc1ccccc1Cl</smiles>

2-(2-(2-chlorophenoxy)phenyl)-2-oxoacetic acid 1c According to procedure A, 1c was obtained as a pale yellow solid (1.42 g, 34\%, 2 steps) after recrystallization in PE / $\mathrm{EA}=30: 1 ;{ }^{1} \mathrm{H} \mathrm{NMR}\left(500 \mathrm{MHz}, \mathrm{CDCl}_{3}\right) \delta 8.96(\mathrm{br}, 1 \mathrm{H}), 7.96(\mathrm{dd}, J=7.8,1.7 \mathrm{~Hz}, 1 \mathrm{H})$, $7.45(\mathrm{dd}, J=8.0,1.6 \mathrm{~Hz}, 1 \mathrm{H}), 7.29-7.19(\mathrm{~m}, 2 \mathrm{H}), 7.19-7.13(\mathrm{~m}, 1 \mathrm{H}), 7.05(\mathrm{~d}, J=8.1$ 
$\mathrm{Hz}, 1 \mathrm{H}), 6.73(\mathrm{~d}, J=8.3 \mathrm{~Hz}, 1 \mathrm{H}) ;{ }^{13} \mathrm{C} \mathrm{NMR}\left(126 \mathrm{MHz}, \mathrm{CDCl}_{3}\right) \delta 185.25,167.48$, $157.81,150.76,136.29,131.26,131.16,128.40,126.51,126.24,123.83,123.70$, 121.46, 116.86; IR (neat): 3597, 3074, 1726, 1678, 1600, 1474, 1446, 1264, 1239, 1061, 981, 879, 753, $652 \mathrm{~cm}^{-1}$; HRMS-ESI (m/z): $[\mathrm{M}-\mathrm{H}+2 \mathrm{Na}]^{+}$calc'd for $\mathrm{C}_{14} \mathrm{H}_{8} \mathrm{ClNa}_{2} \mathrm{O}_{4}$ 320.9901, found 320.9903 .<smiles>O=C(O)c1ccccc1Oc1ccccc1Br</smiles>

2-(2-(2-bromophenoxy)phenyl)-2-oxoacetic acid 1d. According to procedure A, 1d was obtained as a pale yellow solid (1.60 g, 50\%, 2 steps) after recrystallization in PE / $\mathrm{EA}=30: 1 ;{ }^{1} \mathrm{H} \mathrm{NMR}\left(500 \mathrm{MHz}, \mathrm{CDCl}_{3}\right) \delta 7.95(\mathrm{dd}, J=7.8,1.7 \mathrm{~Hz}, 1 \mathrm{H}), 7.62(\mathrm{dd}, J=$ 8.0, 1.5 Hz, 1H), $7.52(\mathrm{ddd}, J=8.6,7.4,1.7 \mathrm{~Hz}, 1 \mathrm{H}), 7.40-7.20(\mathrm{br}, 1 \mathrm{H}), 7.28(\mathrm{td}, J=$ 8.1, $1.5 \mathrm{~Hz}, 1 \mathrm{H}), 7.22(\mathrm{~m}, 1 \mathrm{H}), 7.08(\mathrm{td}, J=7.9,1.4 \mathrm{~Hz}, 1 \mathrm{H}), 7.03(\mathrm{dd}, \mathrm{J}=8.1,1.4 \mathrm{~Hz}$, $1 \mathrm{H}), 6.73(\mathrm{~d}, J=8.4 \mathrm{~Hz}, 1 \mathrm{H}) ;{ }^{13} \mathrm{C} \mathrm{NMR}\left(126 \mathrm{MHz}, \mathrm{CDCl}_{3}\right) \delta 185.66,166.83,157.76$, $152.16,136.18,134.19,131.32,129.14,126.48,124.08,123.73,121.36,117.15$, 115.44; IR (neat): 3066, 1724, 1679, 1599, 1575, 1469, 1442, 1469, 1442, 1261, 1235 , 1210, 1157, 1107, 1045, 980, 876, 750, $656 \mathrm{~cm}^{-1}$; HRMS-ESI (m/z): $\left[\mathrm{M}+\mathrm{NH}_{4}\right]^{+}$calc'd for $\mathrm{C}_{14} \mathrm{H}_{13} \mathrm{O}_{4} \mathrm{NBr}$ 338.0022, found 338.0007.<smiles>O=C(O)C(=O)c1ccccc1Oc1ccc([N+](=O)[O-])cc1</smiles>

2-(2-(4-nitrophenoxy)phenyl)-2-oxoacetic acid $1 \mathrm{~h}$ According to procedure B, $\mathbf{1 h}$ was obtained as a pale yellow solid (1.91 g, 66\%, 2 steps) after recrystallization in $\mathrm{PE} / \mathrm{EA}=20: 1 .{ }^{1} \mathrm{H} \mathrm{NMR}\left(500 \mathrm{MHz}, \mathrm{CDCl}_{3}\right) \delta 8.20(\mathrm{~d}, J=9.2 \mathrm{~Hz}, 2 \mathrm{H}), 8.00(\mathrm{dd}, J=$ 7.9, 1.7 Hz, 1H), 7.66 (ddd, $J=9.1,7.4,1.7 \mathrm{~Hz}, 1 \mathrm{H}), 7.39-7.36(\mathrm{~m}, 1 \mathrm{H}), 7.09$ (d, $J=9.2$ $\mathrm{Hz}, 2 \mathrm{H}), 7.04$ (d, J=7.8 Hz, 1H), 6.46 (br, 1H); ${ }^{13} \mathrm{C} \mathrm{NMR}\left(126 \mathrm{MHz}, \mathrm{CDCl}_{3}\right) \delta 185.12$, $166.10,161.22$, 155.81, 143.90, 136.50, 131.70, 126.18, 125.74, 125.68, 120.15, 
118.36; IR (neat): 3112, 3081, 1724, 1686, 1613, 1599, 1577, 1517, 1478, 1455, 1345 , 1241, 1163, 1110, 981, 880, 849, 750, 685, $644 \mathrm{~cm}^{-1}$; HRMS-ESI (m/z): $[\mathrm{M}+\mathrm{H}]^{+}$calc'd for $\mathrm{C}_{14} \mathrm{H}_{10} \mathrm{O}_{6} \mathrm{~N} 288.0503$, found 288.0502.<smiles>COc1cccc(OC)c1Oc1ccccc1C(=O)C(=O)O</smiles>

2-(2-(2,6-dimethoxyphenoxy)phenyl)-2-oxoacetic acid 1i According to procedure A, 1i was obtained as a brown solid (1.01 g, 36\%, 2 steps) after recrystallization in PE / $\mathrm{EA}=20: 1 .{ }^{1} \mathrm{H}$ NMR $(500 \mathrm{MHz}$, Chloroform- $d$ ) $\delta 8.92(\mathrm{br}, 1 \mathrm{H}), 7.88(\mathrm{dd}, J=7.8,1.7$ Hz, 1H), 7.41 (ddd, $J=8.7,7.4,1.7 \mathrm{~Hz}, 1 \mathrm{H}), 7.14$ (t, $J=8.4 \mathrm{~Hz}, 1 \mathrm{H}), 7.07$ (t, $J=7.5$ $\mathrm{Hz}, 1 \mathrm{H}), 6.60(\mathrm{dd}, J=8.2,6.0 \mathrm{~Hz}, 3 \mathrm{H}), 3.71(\mathrm{~s}, 6 \mathrm{H}) ;{ }^{13} \mathrm{C} \mathrm{NMR}\left(126 \mathrm{MHz}, \mathrm{CDCl}_{3}\right) \delta$ $186.09,168.31,159.40,153.35,136.00,131.19,130.70,126.32,122.43,122.01$, 114.28, 105.71, 56.37; IR (neat): 3097, 3003, 2942, 2840, 1726, 1675, 1599, 1480, 1304, 1259, 1230, 1155, 1110, 980, 875, 756, $735 \mathrm{~cm}^{-1}$; HRMS-ESI (m/z): $[\mathrm{M}+\mathrm{H}]^{+}$ calc'd for $\mathrm{C}_{16} \mathrm{H}_{15} \mathrm{O}_{6}$ 303.0863, found 303.0852.<smiles>CCCc1cccc(C(C)C)c1Oc1ccccc1C(=O)C(=O)O</smiles>

2-(2-(2,6-diisopropylphenoxy)phenyl)-2-oxoacetic acid 1j According to procedure A, 1j was obtained as pale yellow solid (1.25 g, 38\%, 2 steps) after flash chromatography (PE / EA=20:1 to 10:1 to 1:1). $\left.{ }^{1} \mathrm{H} \mathrm{NMR} \mathrm{(500} \mathrm{MHz,} \mathrm{CDCl}_{3}\right) \delta 9.97(\mathrm{br}$, 1H), $7.97(\mathrm{dd}, J=7.8,1.7 \mathrm{~Hz}, 1 \mathrm{H}), 7.42(\mathrm{ddd}, J=8.6,7.3,1.8 \mathrm{~Hz}, 1 \mathrm{H}), 7.30-7.25(\mathrm{~m}$, 1H), 7.19 (d, $J=7.7 \mathrm{~Hz}, 2 \mathrm{H}), 7.08$ (t, $J=8.0 \mathrm{~Hz}, 1 \mathrm{H}), 6.43$ (d, $J=8.0 \mathrm{~Hz}, 1 \mathrm{H}), 2.94$ (p, $J=6.9 \mathrm{~Hz}, 2 \mathrm{H}), 1.04(\mathrm{dd}, J=11.2,6.9 \mathrm{~Hz}, 12 \mathrm{H}) ;{ }^{13} \mathrm{C} \mathrm{NMR}\left(126 \mathrm{MHz}, \mathrm{CDCl}_{3}\right) \delta$ $185.21,169.44,160.08,147.03,141.72,136.60,131.27,126.84,124.91,122.00$, 121.23, 113.70, 27.00, 24.43, 22.63; IR (neat): 3069, 2965, 2929, 2869, 1722, 1679, 1598, 1475, 1458, 1266, 1228, 1208, 1168, 1156, 981, $754 \mathrm{~cm}^{-1}$; HRMS-ESI (m/z): $[\mathrm{M}+2 \mathrm{Na}-\mathrm{H}]^{+}$calc'd for $\mathrm{C}_{20} \mathrm{H}_{22} \mathrm{Na}_{2} \mathrm{O}_{4}$ 371.1230, found 371.1237. 
<smiles>Cc1ccc(Oc2ccccc2C(=O)C(=O)O)c(C)c1</smiles>

2-(2-(2,4-dimethylphenoxy)phenyl)-2-oxoacetic acid 1k According to procedure A, $1 \mathbf{k}$ was obtained as pale yellow solid (1.36 g, 50\%, 2 steps) after flash chromatography (PE / EA=20:1 to 10:1 to $1: 1) ;{ }^{1} \mathrm{H} \mathrm{NMR}\left(500 \mathrm{MHz}, \mathrm{CDCl}_{3}\right) \delta 10.15$ (br, 1H), $7.92(\mathrm{dd}, J=7.8,1.6 \mathrm{~Hz}, 1 \mathrm{H}), 7.51-7.42(\mathrm{~m}, 1 \mathrm{H}), 7.13(\mathrm{t}, J=7.5 \mathrm{~Hz}, 1 \mathrm{H})$, $7.04(\mathrm{~s}, 1 \mathrm{H}), 6.97(\mathrm{~d}, J=8.1 \mathrm{~Hz}, 1 \mathrm{H}), 6.81(\mathrm{~d}, J=8.2 \mathrm{~Hz}, 1 \mathrm{H}), 6.65(\mathrm{~d}, J=8.4 \mathrm{~Hz}, 1 \mathrm{H})$, $2.33(\mathrm{~s}, 3 \mathrm{H}), 2.11(\mathrm{~s}, 3 \mathrm{H}) ;{ }^{13} \mathrm{C} \mathrm{NMR}\left(126 \mathrm{MHz}, \mathrm{CDCl}_{3}\right) \delta 185.49,168.61,159.28$, $150.38,136.41,135.09,132.44,130.95,130.13,128.10,123.06,122.54,120.15$, 115.87, 20.92, 15.90. IR (neat): 3083, 3017, 2919, 1724, 1677, 1597, 1498, 1475, 1253, $1224,1197,1156,980,858,827,752$,

$\mathrm{cm}^{-1}$; HRMS-ESI (m/z): $[\mathrm{M}+2 \mathrm{Na}-\mathrm{H}]^{+}$calc'd for $\mathrm{C}_{16} \mathrm{H}_{13} \mathrm{Na}_{2} \mathrm{O}_{4}$ 315.0604, found 315.0608 .<smiles>COc1ccc2ccccc2c1Oc1ccccc1C(=O)C(=O)O</smiles>

2-(2-((2-methoxynaphthalen-1-yl)oxy)phenyl)-2-oxoacetic acid 11 According to procedure A, 11 was obtained as a brown solid (1.07 g, 34\%, 2 steps) after flash chromatography (PE / EA=10:1 to 3:1 to EA); ${ }^{1} \mathrm{H}$ NMR (500 MHz, DMSO- $\left.d_{6}\right) \delta 7.98$ $7.96(\mathrm{~m}, 2 \mathrm{H}), 7.89(\mathrm{dd}, J=7.8,1.5 \mathrm{~Hz}, 1 \mathrm{H}), 7.81(\mathrm{~d}, J=8.6 \mathrm{~Hz}, 1 \mathrm{H}), 7.62(\mathrm{~d}, J=9.1 \mathrm{~Hz}$, 1H), 7.50 - $7.47(\mathrm{~m}, 2 \mathrm{H}), 7.43(\mathrm{t}, J=7.9 \mathrm{~Hz}, 1 \mathrm{H}), 7.18(\mathrm{t}, J=7.5 \mathrm{~Hz}, 1 \mathrm{H}), 3.84(\mathrm{~s}, 3 \mathrm{H})$; ${ }^{13} \mathrm{C}$ NMR (126 MHz, DMSO-d $) \delta$ 187.99, 166.86, 158.56, 148.36, 136.43, 133.94, $130.28,129.16,127.99,127.68,127.19,127.08,124.42,122.25,121.74,120.33$, 115.35, 113.64, 56.68; IR (neat): 3164, 3069, 3011, 2939, 2844, 1724, 1674, 1598, 1508, 1476, 1456, 1376, 1277, 1257, 1222, 1088, 807, $752 \mathrm{~cm}^{-1}$; HRMS-ESI (m/z): $[\mathrm{M}+\mathrm{H}]^{+}$calc'd for $\mathrm{C}_{19} \mathrm{H}_{15} \mathrm{O}_{5} \quad 323.0914$, found 323.0899 . 
<smiles>O=C(O)c1ccccc1Oc1cccc2cccnc12</smiles>

2-oxo-2-(2-(quinolin-8-yloxy)phenyl)acetic acid 1m According to procedure A, 1m was obtained as a brown solid (1.50 g, 52\%, 2 steps) after washed by DCM and $\mathrm{H}_{2} \mathrm{O}$ instead of treated by $1 \mathrm{M} \mathrm{HCl} ;{ }^{1} \mathrm{H}$ NMR $\left(500 \mathrm{MHz}, \mathrm{DMSO}-d_{6}\right) \delta 8.87(\mathrm{dd}, \mathrm{J}=4.2,1.7$ $\mathrm{Hz}, 1 \mathrm{H}), 8.48(\mathrm{dd}, \mathrm{J}=8.4,1.6 \mathrm{~Hz}, 1 \mathrm{H}), 7.93(\mathrm{dd}, \mathrm{J}=8.3,1.1 \mathrm{~Hz}, 1 \mathrm{H}), 7.89$ (dd, J = 7.8, $1.7 \mathrm{~Hz}, 1 \mathrm{H}), 7.66(\mathrm{t}, \mathrm{J}=7.9 \mathrm{~Hz}, 1 \mathrm{H}), 7.62(\mathrm{dd}, \mathrm{J}=8.3,4.2 \mathrm{~Hz}, 1 \mathrm{H}), 7.54$ (ddd, J = 8.5, 7.3, $1.8 \mathrm{~Hz}, 1 \mathrm{H}), 7.43(\mathrm{dd}, \mathrm{J}=7.6,1.2 \mathrm{~Hz}, 1 \mathrm{H}), 7.28-7.22(\mathrm{~m}, 1 \mathrm{H}), 6.62(\mathrm{~d}, \mathrm{~J}=8.1 \mathrm{~Hz}$, $1 \mathrm{H}) ;{ }^{13} \mathrm{C}$ NMR $\left(126 \mathrm{MHz}, \mathrm{DMSO}-d_{6}\right) \delta 188.05,166.48,159.21,150.59,150.38,140.71$, $136.55,136.29$, 130.15, 129.64, 127.01, 125.46, 123.36, 123.01, 122.40, 120.16, 117.09; IR (neat): 3077, 2898, 2758, 1683, 1600, 1553, 1420, 1380, 1285, 1215, 1181 , 1133, 1100, 893, 839, 759, 736, 684, $669 \mathrm{~cm}^{-1}$; HRMS-ESI (m/z): $[\mathrm{M}+\mathrm{H}]^{+}$calc'd for $\mathrm{C}_{17} \mathrm{H}_{11} \mathrm{NO}_{4}$ 294.0761, found 294.0763.<smiles>Cc1cc(C)c(Oc2ccc(-c3ccccc3)cc2C(=O)C(=O)O)c(C)c1</smiles>

2-(4-(mesityloxy)-[1,1'-biphenyl]-3-yl)-2-oxoacetic acid 10 According to procedure A, 10 was obtained as a pale yellow solid (1.43 g, 65\%, 2 steps) after flash chromatography (PE / EA=10:1 to 1:1); ${ }^{1} \mathrm{H}$ NMR (500 MHz, DMSO- $\left.d_{6}\right) \delta 8.11$ (d, $J=$ $2.5 \mathrm{~Hz}, 1 \mathrm{H}), 7.86(\mathrm{dd}, J=8.7,2.5 \mathrm{~Hz}, 1 \mathrm{H}), 7.64$ (d, $J=7.4 \mathrm{~Hz}, 2 \mathrm{H}), 7.46$ (t, $J=7.7$ $\mathrm{Hz}, 2 \mathrm{H}), 7.37$ (t, $J=7.4 \mathrm{~Hz}, 1 \mathrm{H}), 7.00$ (s, 2H), 6.50 (d, $J=8.7 \mathrm{~Hz}, 1 \mathrm{H}), 2.27$ (s, 3H), $2.03(\mathrm{~s}, 6 \mathrm{H}) ;{ }^{13} \mathrm{C}$ NMR $\left(126 \mathrm{MHz}, \mathrm{DMSO}-d_{6}\right) \delta 187.98,167.38,157.74,147.55$, $138.75,135.53,135.42,134.51,130.57,130.19,129.49,128.55,128.03,126.85$, 122.03, 114.02, 20.81, 16.05; IR (neat): 2918, 1720, 1676, 1604, 1474, 1451, 1261, 1232, 1197, 1178, 1142, 760, $697 \mathrm{~cm}^{-1}$; HRMS-ESI $(\mathrm{m} / \mathrm{z}):[\mathrm{M}+2 \mathrm{Na}-\mathrm{H}]^{+}$calc'd for $\mathrm{C}_{23} \mathrm{H}_{19} \mathrm{Na}_{2} \mathrm{O}_{4}$ 405.1073, found 405.1078. 
<smiles>Cc1cc(C)c(Oc2ccc(C#CC(C)(C)C)cc2C(=O)C(=O)O)c(C)c1</smiles>

2-(5-(3,3-dimethylbut-1-yn-1-yl)-2-(mesityloxy)phenyl)-2-oxoacetic acid 1p According to procedure A, 1p was obtained as a pale yellow solid (1.43 g, 33\%, 2 steps) after flash chromatography (PE / EA=10:1 to 1:1); ${ }^{1} \mathrm{H}$ NMR (500 MHz, $\left.\mathrm{CDCl}_{3}\right)$ $\delta 7.97(\mathrm{~s}, 1 \mathrm{H}), 7.80(\mathrm{br}, 1 \mathrm{H}), 7.39(\mathrm{~d}, J=8.7 \mathrm{~Hz}, 1 \mathrm{H}), 6.88(\mathrm{~s}, 2 \mathrm{H}), 6.34(\mathrm{~d}, J=8.7 \mathrm{~Hz}$, 1H), 2.31 (s, 3H), 1.99 (s, 6H), 1.29 (s, 9H); $\left.{ }^{13} \mathrm{C} \mathrm{NMR} \mathrm{(126} \mathrm{MHz,} \mathrm{CDCl}_{3}\right) \delta 184.90$, $168.07,157.84,147.40,139.34,135.66,134.42$, 130.76, 130.02, 121.45, 118.21, 113.23, 98.98, 77.26, 31.08, 28.06, 20.94, 16.07; IR (neat): 3036, 2969, 2924, 2867, 2245, 1726, 1678, 1603, 1474, 1398, 1265, 1234, 1195, 1160, 1142, 1023, 855, 828, $738 \mathrm{~cm}^{-1}$; HRMS-ESI (m/z): $[\mathrm{M}+2 \mathrm{Na}-\mathrm{H}]^{+}$calc'd for $\mathrm{C}_{23} \mathrm{H}_{23} \mathrm{Na}_{2} \mathrm{O}_{4} 409.1386$, found 409.1388 .<smiles>COc1ccc(C(=O)C(=O)O)c(Oc2c(C)cc(C)cc2C)c1</smiles>

2-(2-(mesityloxy)-4-methoxyphenyl)-2-oxoacetic acid 1q According to procedure A, $1 q$ was obtained as a pale yellow solid (1.94 g, 64\%, 2 steps) after recrystallization $(\mathrm{PE} / \mathrm{EA}=20: 1) ;{ }^{1} \mathrm{H} \mathrm{NMR}\left(500 \mathrm{MHz}, \mathrm{CDCl}_{3}\right) \delta 9.11(\mathrm{br}, 1 \mathrm{H}), 7.99(\mathrm{~d}, J=8.8 \mathrm{~Hz}, 1 \mathrm{H})$, $6.86(\mathrm{~s}, 2 \mathrm{H}), 6.59$ (dd, $J=8.9,2.3 \mathrm{~Hz}, 1 \mathrm{H}), 5.89$ (d, $J=2.3 \mathrm{~Hz}, 1 \mathrm{H}), 3.71(\mathrm{~s}, 3 \mathrm{H}), 2.30$ (s, 3H), 2.02 (s, 6H); ${ }^{13} \mathrm{C}$ NMR $\left(126 \mathrm{MHz}, \mathrm{CDCl}_{3}\right) \delta$ 183.77, 169.44, 167.10, 161.13, $147.33,135.49,133.51,130.73,129.95,114.64,107.62$, 99.00, 55.80, 20.94, 16.06; IR (neat): 3011, 2918, 2842, 1708, 1647, 1597, 1572, 1441, 1280, 1198, 1165, 1140, 1103, 984, 959, 851, $842 \mathrm{~cm}^{-1}$; HRMS-ESI $(\mathrm{m} / \mathrm{z}):[\mathrm{M}-\mathrm{H}+2 \mathrm{Na}]^{+}$calc'd for $\mathrm{C}_{18} \mathrm{H}_{17} \mathrm{Na}_{2} \mathrm{O}_{5}$ 359.0866, found 359.0869. 
<smiles>Cc1cc(C)c(Oc2cc(Cl)ccc2C(=O)O)c(C)c1</smiles>

2-(4-chloro-2-(mesityloxy)phenyl)-2-oxoacetic acid 1r According to procedure A, 1r was obtained as a pale yellow solid (1.96 g, 63\%, 2 steps) after recrystallization $(\mathrm{PE} / \mathrm{EA}=30: 1) ;{ }^{1} \mathrm{H} \mathrm{NMR}\left(500 \mathrm{MHz}, \mathrm{CDCl}_{3}\right) \delta 8.94(\mathrm{br}, 1 \mathrm{H}), 7.90(\mathrm{~d}, J=8.4 \mathrm{~Hz}, 1 \mathrm{H})$, $7.06(\mathrm{~d}, J=8.4 \mathrm{~Hz}, 1 \mathrm{H}), 6.89(\mathrm{~s}, 2 \mathrm{H}), 6.42(\mathrm{~s}, 1 \mathrm{H}), 2.33(\mathrm{~s}, 3 \mathrm{H}), 2.01(\mathrm{~s}, 6 \mathrm{H}) ;{ }^{13} \mathrm{C} \mathrm{NMR}$ $\left(126 \mathrm{MHz}, \mathrm{CDCl}_{3}\right) \delta 184.39,168.40,159.34,147.09,143.18,135.99,132.41,130.57$, 130.19, 122.61, 120.04, 113.62, 20.96, 16.07; IR (neat): 3091, 3014, 2917, 2855, 1722, 1677, 1569, 1474, 1401, 1227, 1193, 1137, 1085, 982, 913, 854, $773 \mathrm{~cm}^{-1}$; HRMS-ESI (m/z): [M-H+2Na] $]^{+}$calc'd for $\mathrm{C}_{17} \mathrm{H}_{14} \mathrm{ClNa}_{2} \mathrm{O}_{4}$ 363.0371, found 363.0372.<smiles>Cc1cc(C)c(Oc2cc(Br)ccc2C(=O)C(=O)O)c(C)c1</smiles>

2-(4-bromo-2-(mesityloxy)phenyl)-2-oxoacetic acid 1s According to procedure A, 1s was obtained as a pale yellow solid (1.54 g, 43\%, 2 steps) after recrystallization $(\mathrm{PE} / \mathrm{EA}=30: 1) ;{ }^{1} \mathrm{H} \mathrm{NMR}\left(500 \mathrm{MHz}, \mathrm{CDCl}_{3}\right) \delta 9.71(\mathrm{br}, 1 \mathrm{H}), 7.80(\mathrm{~d}, J=8.4 \mathrm{~Hz}, 1 \mathrm{H})$, $7.23(\mathrm{dd}, J=8.4,1.7 \mathrm{~Hz}, 1 \mathrm{H}), 6.89(\mathrm{~s}, 2 \mathrm{H}), 6.58(\mathrm{~d}, J=1.7 \mathrm{~Hz}, 1 \mathrm{H}), 2.32$ (s, 3H), 2.01 $(\mathrm{s}, 6 \mathrm{H}) ;{ }^{13} \mathrm{C} \mathrm{NMR}\left(126 \mathrm{MHz}, \mathrm{CDCl}_{3}\right) \delta 184.70,168.49,159.05,147.06,135.96,132.38$, 131.69, 130.55, 130.18, 125.55, 120.49, 116.52, 20.96, 16.08; IR (neat): 3080, 2918, 2864, 1719, 1673, 1584, 1567, 1471, 1399, 1251, 1221, 1202, 982, 893, 855, 735, 714 $\mathrm{cm}^{-1}$; HRMS-ESI (m/z): [M-H+2Na $]^{+}$calc'd for $\mathrm{C}_{17} \mathrm{H}_{14} \mathrm{BrNa}_{2} \mathrm{O}_{4}$ 408.9865, found 408.9869 .<smiles>Cc1cc(C)c(Oc2cc(C#N)ccc2C(=O)C(=O)O)c(C)c1</smiles>

2-(4-cyano-2-(mesityloxy)phenyl)-2-oxoacetic acid 1t According to procedure A, 1t 
was obtained as a pale yellow solid (1.30 g, 50\%, 2 steps) after recrystallization (PE $/ \mathrm{EA}=15: 1) ;{ }^{1} \mathrm{H}$ NMR $\left(500 \mathrm{MHz}, \mathrm{DMSO}-d_{6}\right) \delta 7.97(\mathrm{~d}, J=8.0 \mathrm{~Hz}, 1 \mathrm{H}), 7.61(\mathrm{~d}, J=$ $7.9 \mathrm{~Hz}, 1 \mathrm{H}), 7.00(\mathrm{~s}, 2 \mathrm{H}), 6.74(\mathrm{~s}, 1 \mathrm{H}), 2.27$ (s, 3H), $1.99(\mathrm{~s}, 6 \mathrm{H}) ;{ }^{13} \mathrm{C}$ NMR (126 MHz, DMSO- $\left.d_{6}\right) \delta 188.25,166.10,156.99,146.66,135.37,131.57,130.00,129.88,126.52$, 125.66, 117.42, 117.24, 116.11, 20.38, 15.50; IR (neat): 2965, 2923, 2235, 1736, 1686, 1604, 1564, 1480, 1407, 1257, 1159, 1152, 1138, 984, 856, $799 \mathrm{~cm}^{-1}$; HRMS-ES (m/z): $[\mathrm{M}-\mathrm{H}+2 \mathrm{Na}]^{+}$calc'd for $\mathrm{C}_{17} \mathrm{H}_{14} \mathrm{BrNa}_{2} \mathrm{O}_{4}$ 354.0713, found 354.0718.<smiles>Cc1cc(C)c(Oc2ccc(C(F)(F)F)cc2C(=O)C(=O)O)c(C)c1</smiles>

2-(2-(mesityloxy)-4-(trifluoromethyl)phenyl)-2-oxoacetic acid 1u According to procedure A, 1u was obtained as a pale yellow solid (0.764 g, 24\%, 2 steps) after flash chromatography (PE / EA=10:1 to $1: 1) .{ }^{1} \mathrm{H} \mathrm{NMR}\left(500 \mathrm{MHz}, \mathrm{CDCl}_{3}\right) \delta 8.22(\mathrm{~d}, J$ $=2.1 \mathrm{~Hz}, 1 \mathrm{H}), 7.64(\mathrm{dd}, J=8.8,2.0 \mathrm{~Hz}, 1 \mathrm{H}), 6.91(\mathrm{~s}, 2 \mathrm{H}), 6.56(\mathrm{~d}, J=8.8 \mathrm{~Hz}, 1 \mathrm{H}), 5.80$ (br, 1H), 2.32 (s, 3H), $2.02(\mathrm{~s}, 6 \mathrm{H}) .{ }^{13} \mathrm{C} \mathrm{NMR}\left(126 \mathrm{MHz}, \mathrm{CDCl}_{3}\right) \delta$ 184.89, 167.09, $160.79,147.15,136.22,133.11\left(\mathrm{q},{ }^{3} J_{\mathrm{C}-\mathrm{F}}=3.4 \mathrm{~Hz}\right), 130.58,130.19,128.94\left(\mathrm{q},{ }^{3} J_{\mathrm{C}-\mathrm{F}}=3.4\right.$ $\mathrm{Hz}), 126.87,124.99,124.59\left(\mathrm{q},{ }^{2} J_{\mathrm{C}-\mathrm{F}}=33.9 \mathrm{~Hz}\right), 123.62\left(\mathrm{q},{ }^{1} J_{\mathrm{C}-\mathrm{F}}=271.2 \mathrm{~Hz}\right), 121.89$, 113.85, 20.92, 16.06; ${ }^{19} \mathrm{~F}$ NMR (376 MHz, $\left.\mathrm{CDCl}_{3}\right) \delta$-63.10; IR (neat): 3567, 2924, $1726,1686,1617,1497,1480,1443,1334,1314,1268,1245,1195,1130,1075,856$, 834, $660 \mathrm{~cm}^{-1}$; HRMS-ESI (m/z): $[\mathrm{M}+\mathrm{H}]^{+}$calc'd for $\mathrm{C}_{18} \mathrm{H}_{16} \mathrm{O}_{4} \mathrm{~F}_{3}$ 353.0995, found 353.0993 .<smiles>CCOC(=O)c1ccc(I)cc1</smiles><smiles>CCOC(=O)c1ccc(Oc2ccccc2C(C)=O)cc1</smiles><smiles>CCOC(=O)c1ccc(Oc2ccccc2C(=O)C(=O)O)cc1</smiles>

2-(2-(4-(ethoxycarbonyl)phenoxy)phenyl)-2-oxoacetic acid 1f 
Following the literature procedure ${ }^{8}$, an oven-dried $100 \mathrm{~mL}$ round-bottomed flask equipped with a magnetic stir bar was added copper(I) iodide $(95.5 \mathrm{mg}, 0.5 \mathrm{mmol}, 5$ mol\%), picolinic acid (123 mg, $1.0 \mathrm{mmol}, 10 \mathrm{~mol} \%)$, aryl iodide (2.76 g, $10 \mathrm{mmol})$, $\operatorname{ArOH}(1.63 \mathrm{~g}, 12 \mathrm{mmol})$, and $\mathrm{K}_{3} \mathrm{PO}_{4}(4.24 \mathrm{~g}, 20 \mathrm{mmol})$. The flask was then evacuated and backfilled with $\mathrm{N}_{2}$ sequence for 3 times, followed by dimethyl sulfoxide $(25 \mathrm{~mL})$ addition by syringe. The flask was placed in a preheated oil bath at $80{ }^{\circ} \mathrm{C}$, and the reaction mixture was stirred vigorously for $24 \mathrm{~h}$. The reaction was cooled to room temperature, $\mathrm{H}_{2} \mathrm{O}(50 \mathrm{~mL})$ was added and the mixture was extracted by ethyl acetate (30 $\mathrm{mL}^{*} 3$ ). The combined organic layer was dried over $\mathrm{Na}_{2} \mathrm{SO}_{4}$ and filtered and concentrated. The crude product was purified by flash chromatography. The subsequent selenium dioxide oxidation is same as procedure $A$ to afford $\mathbf{1 f}$ as a pale yellow solid $\left(0.547 \mathrm{~g}, 17 \%, 2\right.$ steps) after recrystallization in PE / EA $=20: 1 ;{ }^{1} \mathrm{H}$ NMR (500 MHz, DMSO-d6) $\delta 7.98(\mathrm{~d}, J=8.9 \mathrm{~Hz}, 2 \mathrm{H}), 7.90(\mathrm{dd}, J=7.8,1.7 \mathrm{~Hz}, 1 \mathrm{H})$, $7.74-7.70(\mathrm{~m}, 1 \mathrm{H}), 7.41-7.38(\mathrm{~m}, 1 \mathrm{H}), 7.11-7.09(\mathrm{~m}, 3 \mathrm{H}), 4.29(\mathrm{q}, J=7.1 \mathrm{~Hz}, 2 \mathrm{H})$, $1.29(\mathrm{t}, J=7.1 \mathrm{~Hz}, 3 \mathrm{H}) ;{ }^{13} \mathrm{C}$ NMR (500 MHz, DMSO-d6) $\delta$ 187.82, 166.10, 165.07, 159.73, 155.87, 136.41, 131.46, 130.57, 125.46, 125.38, 125.08, 119.94, 118.38, 60.70, 14.20; IR (neat): 3073, 2985, 1741, 1712, 1688, 1602, 1504, 1478, 1456, 1370, 1280, 1241, 1163, 1105, 1015, 980, 874, $766 \mathrm{~cm}^{-1}$; HRMS-ESI $(\mathrm{m} / \mathrm{z}):[\mathrm{M}+\mathrm{Na}]^{+}$calc'd for $\mathrm{C}_{17} \mathrm{H}_{14} \mathrm{NaO}_{6}$ 337.0683, found 337.0685.<smiles>CCOC(=O)c1ccc(I)cc1</smiles><smiles>CCOc1ccc(Oc2ccc(C)cc2C(C)=O)cc1</smiles><smiles>CCOC(=O)c1ccc(Oc2ccccc2C(=O)C(=O)O)cc1</smiles>

\section{2-(2-(4-(ethoxycarbonyl)phenoxy)-5-methylphenyl)-2-oxoacetic acid 1n}

Following the literature procedure ${ }^{8}$, An oven-dried $100 \mathrm{~mL}$ round-bottomed flask equipped with a magnetic stir bar, copper(I) iodide (190 mg, $1.0 \mathrm{mmol}, 10 \mathrm{~mol} \%)$, picolinic acid (246 mg, $2.0 \mathrm{mmol}, 20 \mathrm{~mol} \%$ ), aryl iodide (2.76 g, $10 \mathrm{mmol}), \mathrm{ArOH}$ (1.63 g, $12 \mathrm{mmol}$ ), and $\mathrm{K}_{3} \mathrm{PO}_{4}(4.24 \mathrm{~g}, 20 \mathrm{mmol})$. The flask was then 
evacuation/backfill with $\mathrm{N}_{2}$ sequence (3 times), followed by dimethyl sulfoxide (25 $\mathrm{mL}$ ) by syringe. The flask was placed in a preheated oil bath at $80{ }^{\circ} \mathrm{C}$, and the reaction mixture was stirred vigorously for $24 \mathrm{~h}$. The reaction was cooled to room temperature, $\mathrm{H}_{2} \mathrm{O}(50 \mathrm{~mL})$ was added and the mixture was extracted by ethyl acetate $\left(30 \mathrm{~mL}^{*} 3\right)$. The combined organic layer was dried over $\mathrm{Na}_{2} \mathrm{SO}_{4}$ and filtered and concentrated. The crude product was purified by flash chromatography.

The same procedure as the step 2 of Procedure A to afford $\mathbf{1 n}$ as pale yellow solid (0.547 g, 17\%, 2 steps) after recrystallization in PE / EA = 20:1; ${ }^{1} \mathrm{H}$ NMR $(500 \mathrm{MHz}$, DMSO-d $) \delta 7.98(\mathrm{~d}, J=8.9 \mathrm{~Hz}, 2 \mathrm{H}), 7.71(\mathrm{~d}, J=1.6 \mathrm{~Hz}, 1 \mathrm{H}), 7.55(\mathrm{dd}, J=8.4,1.7 \mathrm{~Hz}$, 1H), $7.08(\mathrm{~d}, J=8.9 \mathrm{~Hz}, 2 \mathrm{H}), 7.04(\mathrm{~d}, J=8.4 \mathrm{~Hz}, 1 \mathrm{H}), 4.30(\mathrm{q}, J=7.1 \mathrm{~Hz}, 2 \mathrm{H}), 2.37(\mathrm{~s}$, $3 \mathrm{H}), 1.31(\mathrm{t}, J=7.1 \mathrm{~Hz}, 3 \mathrm{H}) ;{ }^{13} \mathrm{C}$ NMR $\left(126 \mathrm{MHz}, \mathrm{DMSO}-d_{6}\right) \delta 187.68,166.09,165.10$, $160.14,153.66,137.11,134.76,131.41,130.30,125.17,125.12,120.25,117.99,60.67$, 20.09, 14.21; IR (neat): 3162, 2984, 1743, 1715, 1684, 1603, 1504, 1488, 1417, 1279, 1245, 1163, 1123, 1109, 1015, 856. 770, 668, 693, $612 \mathrm{~cm}^{-1}$; HRMS-ESI (m/z): $[\mathrm{M}+\mathrm{Na}]^{+}$calc'd for $\mathrm{C}_{18} \mathrm{H}_{16} \mathrm{NaO}_{6} 351.0839$, found 351.0842 .<smiles>COC(OC)c1ccc(Oc2cccc(C(C)(O)O)c2Br)cc1</smiles>

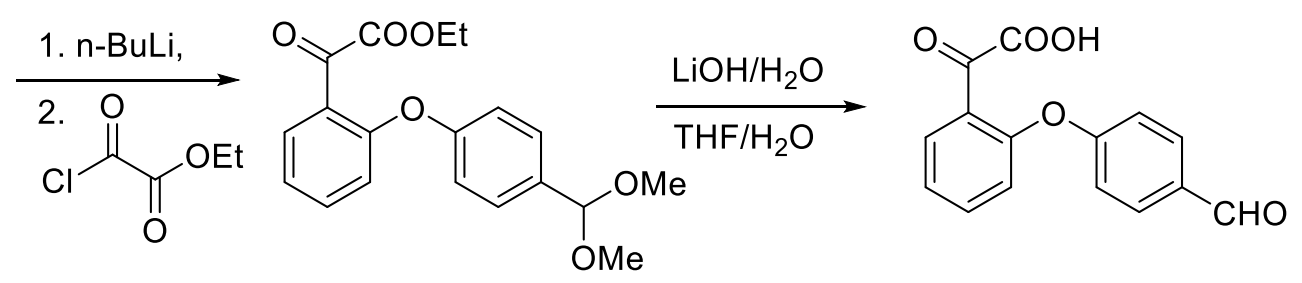

\section{2-(2-(4-formylphenoxy)phenyl)-2-oxoacetic acid $1 \mathrm{~g}$}

The phenol (22 mmol, 1.1 equiv.) and anhydrous $\mathrm{K}_{2} \mathrm{CO}_{3}$ (24 mmol 1.2 equiv.) were added to a solution of 2-fluorobenzaldehyde $(20 \mathrm{mmol})$ in DMA $(0.5 \mathrm{M})$ and the resulting mixture was slowly heated to $170{ }^{\circ} \mathrm{C} .{ }^{5}$ After stirring at that temperature for 4 $h$ and cooling to room temperature the reaction mixture was poured into $\mathrm{H}_{2} \mathrm{O}$ and the aqueous layer was extracted with DCM. After drying over $\mathrm{Na}_{2} \mathrm{SO}_{4}$, filtration and 
concentration in vacuo, the desired 2-phenoxybenzketone was purified by silica gel column chromatography, afforded the product as pale yellow solid, $4.24 \mathrm{~g}, 77 \%$.

2-phenoxybenzaketone (15.4 mmol), TsOH$\cdot \mathrm{H}_{2} \mathrm{O}$ (cat. $\left.40 \mathrm{mg}\right), \mathrm{HC}(\mathrm{OMe})_{3}(40 \mathrm{mmol})$ and $\mathrm{MeOH}(7 \mathrm{~mL})$, After stirring at room temperature for $20 \mathrm{~h}$, the mixture was treated with $\mathrm{NaHCO}_{3}$ and then concentrated in vacuo. The benzaldehyde dimethylacetal was obtained by flash chromatography on silica gel treated with $1 \%$ $\mathrm{NEt}_{3}$ in PE (PE:EA:NEt ${ }_{3}=30: 1: 0.3$ ), afforded the product as a pale yellow liquid, 4.58 $\mathrm{g}, 92 \%$.

A solution of benzaldehyde dimethylacetal $(7 \mathrm{mmol})$ in dry THF $(10 \mathrm{~mL})$ under $\mathrm{N}_{2}$ atmosphere was cooled to $-78{ }^{\circ} \mathrm{C}$, and n-butylithium $(2.5 \mathrm{M}$ in hexane, $3.5 \mathrm{~mL}, 8.7$ $\mathrm{mmol}$ ), after stirring at this temperature for $2 \mathrm{~h}$, the lithium reagent was added into the solution of ethyl chloroglyoxylate $(5.8 \mathrm{mmol})$ in dry THF $(15 \mathrm{~mL})$ under $\mathrm{N}_{2}$ atmosphere at $-78{ }^{\circ} \mathrm{C}$, after stirring at this temperature for $2 \mathrm{~h}, \mathrm{H}_{2} \mathrm{O}$ was added into the mixture at room temperature to quench the reaction, the aqueous layer was extracted with EA. After drying over $\mathrm{Na}_{2} \mathrm{SO}_{4}$, filtration and concentration in vacuo, The desired product was obtained by flash chromatography on silica gel treated with $1 \% \mathrm{NEt}_{3}$ in PE (PE:EA:NEt $3=5: 1: 0.1$ ), afforded the product as a colorless liquid, $0.267 \mathrm{~g}, 13 \%$.

the product in step $3(0.77 \mathrm{mmol})$ and $\mathrm{LiOH} \cdot \mathrm{H}_{2} \mathrm{O}(3.85 \mathrm{mmol}, 5$ equiv. $)$ in $\mathrm{THF} / \mathrm{H}_{2} \mathrm{O}=3: 1(6 \mathrm{~mL})^{9}$, after stirring at room temperature overnight, removed THF in vacuo, the aqueous layer was washed with DCM $\left(5 \mathrm{~mL}^{* 2}\right)$, then treated with $1 \mathrm{M} \mathrm{HCl}$ to $\mathrm{pH}=1$, extracted with $\mathrm{EA}(10 \mathrm{~mL} * 3)$, the combined organic layer was washed with brine, dried over with $\mathrm{Na}_{2} \mathrm{SO}_{4}$ and concentrated, afforded the product as a white solid, $0.158 \mathrm{~g}, 76 \%$

${ }^{1} \mathrm{H}$ NMR (500 MHz, DMSO-d $\left.d_{6}\right) \delta 9.96(\mathrm{~s}, 1 \mathrm{H}), 7.96(\mathrm{~d}, J=8.8 \mathrm{~Hz}, 2 \mathrm{H}), 7.92(\mathrm{dd}, J=$ 7.8, $1.7 \mathrm{~Hz}, 1 \mathrm{H}), 7.78-7.73(\mathrm{~m}, 1 \mathrm{H}), 7.46-7.41(\mathrm{~m}, 1 \mathrm{H}), 7.19-7.16(\mathrm{~m}, 3 \mathrm{H}),{ }^{13} \mathrm{C} \mathrm{NMR}$ $\left(126 \mathrm{MHz}, \mathrm{DMSO}-d_{6}\right) \delta 191.64,188.05,166.16,160.92,155.39,136.33,132.08$, 131.88, 130.69, 125.71, 125.35, 120.43, 118.49; IR: 3330, 3061, 1736, 1692, 1599, 1578, 1502, 1477, 1237, 1156, 977, 833, 789, $618 \mathrm{~cm}^{-1}$; HRMS-ESI (m/z): $[\mathrm{M}+\mathrm{H}]^{+}$ calc'd for $\mathrm{C}_{15} \mathrm{H}_{11} \mathrm{O}_{5}$ 271.0601, found 271.0593. 


\section{Smiles Rearrangement Product Characterization}

\section{Standard Procedure}
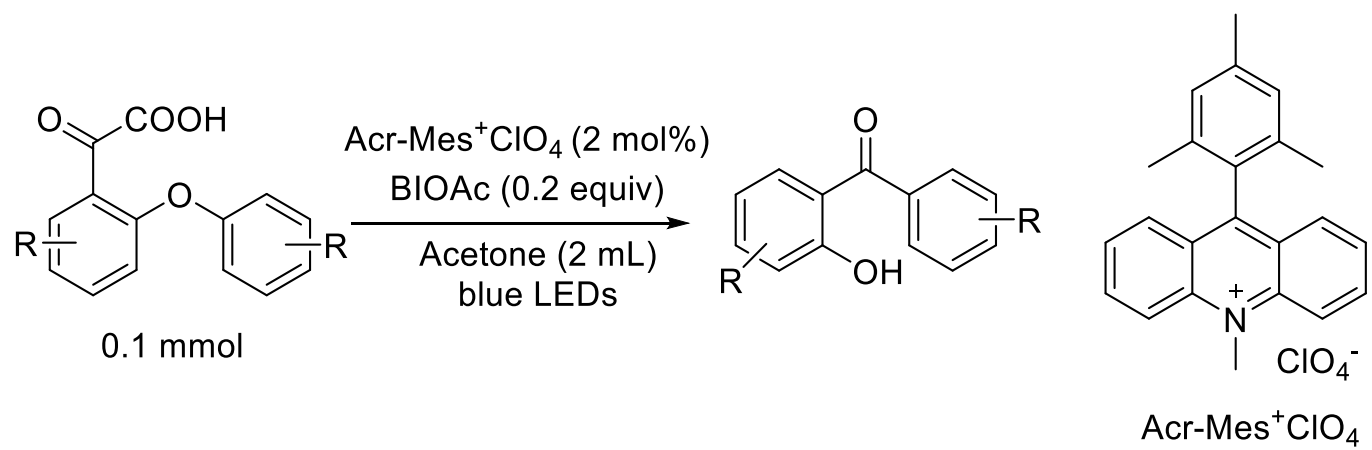

Ketoacid (0.10 mmol), Acr-Mes- $\mathrm{ClO}_{4}(0.8 \mathrm{mg}, 0.002 \mathrm{mmol}, 0.02$ equiv.), and BIOAc (6.1 mg, $0.02 \mathrm{mmol}, 0.2$ equiv.) were placed in a $10 \mathrm{~mL}$ schlenk tube equipped with a magnetic stir bar. After $2.0 \mathrm{~mL}$ acetone (treated by $4 \AA \mathrm{MS}$ ) was added, the reaction mixture was degassed using a freeze-pump-thaw procedure for 3 times and exposed to blue LEDs (two $4 \mathrm{~W}$ LED light bulbs $10 \mathrm{~cm}$ away from the vial, $468 \mathrm{~nm}$ peak wavelength, $25 \mathrm{~nm}$ spectral half-wave width, composed of 55-65 LED units each with $60 \mathrm{~mW}, 3 \mathrm{~V}, 20 \mathrm{~mA}$ ) at room temperature with stirring (typically $12 \mathrm{~h} *$ ). The reaction mixture was concentrated and purified directly by column chromatography to afford the product.

*The heating effect from LED irradiation conditions above was minimal. With 24 hours irradiation, the increase of temperature was less than $3^{\circ} \mathrm{C}$.
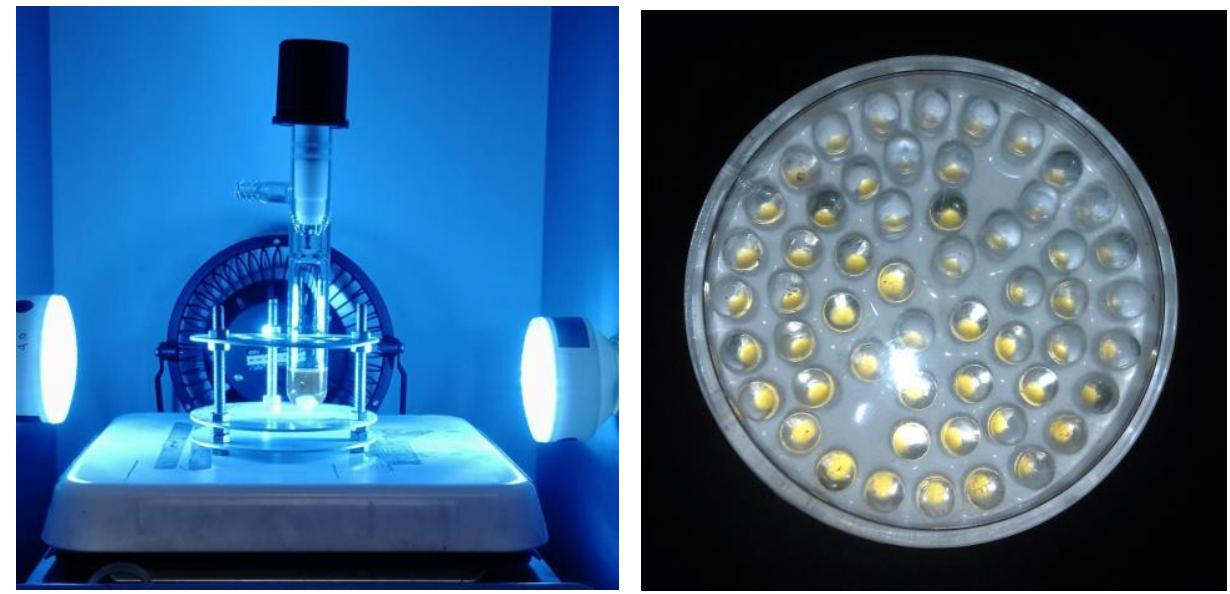
<smiles>Cc1cc(C)c(C(=O)c2ccccc2O)c(C)c1</smiles>

(2-hydroxyphenyl)(mesityl)methanone (CAS: 46863-20-1) $2 \mathbf{a}^{10}$ Following the standard procedure, the reaction of $\mathbf{1 a}$ afforded $\mathbf{2 a}$ as a white solid (20.1 $\mathrm{mg}, 84 \%)$ after flash chromatography: TLC $\mathrm{R}_{\mathrm{f}}=0.35(\mathrm{PE} / \mathrm{EA}=100: 1) ;{ }^{1} \mathrm{H}$ NMR $(500 \mathrm{MHz}$, $\left.\mathrm{CDCl}_{3}\right) \delta 12.34(\mathrm{~s}, 1 \mathrm{H}), 7.48(\mathrm{ddd}, J=8.6,7.3,1.7 \mathrm{~Hz}, 1 \mathrm{H}), 7.18(\mathrm{dd}, J=8.0,1.7 \mathrm{~Hz}$, $1 \mathrm{H}), 7.05(\mathrm{~d}, J=8.4 \mathrm{~Hz}, 1 \mathrm{H}), 6.91(\mathrm{~s}, 2 \mathrm{H}), 6.82-6.74(\mathrm{~m}, 1 \mathrm{H}), 2.34(\mathrm{~s}, 3 \mathrm{H}), 2.12(\mathrm{~s}, 6 \mathrm{H})$; ${ }^{13} \mathrm{C}$ NMR $\left(126 \mathrm{MHz}, \mathrm{CDCl}_{3}\right) \delta 206.78,162.88,138.97,136.88,135.43,133.96$, $132.75,128.33,120.30,119.25,118.27,21.16,19.27$;<smiles>O=C(c1ccccc1F)c1ccccc1F</smiles>

(2-fluorophenyl)(2-hydroxyphenyl)methanone (CAS: $\quad 329235-41-8) \quad$ 2b $^{11}$ Following the standard procedure, irradiation for $16 \mathrm{~h}$, the reaction of $\mathbf{1 b}$ afforded $\mathbf{2 b}$ as a white solid (15.6 mg, 72\%) after flash chromatography: TLC $\mathrm{R}_{\mathrm{f}}=0.60$ (PE / EA $=20: 1) ;{ }^{1} \mathrm{H} \mathrm{NMR}\left(500 \mathrm{MHz}, \mathrm{CDCl}_{3}\right) \delta 11.97(\mathrm{~s}, 1 \mathrm{H}), 7.58-7.49(\mathrm{~m}, 2 \mathrm{H}), 7.47(\mathrm{td}, J=$ 7.5, $1.8 \mathrm{~Hz}, 1 \mathrm{H}), 7.44-7.39(\mathrm{~m}, 1 \mathrm{H}), 7.29$ (td, $J=7.5,0.8 \mathrm{~Hz}, 1 \mathrm{H}), 7.20$ (t, $J=9.0 \mathrm{~Hz}$, 1H), $7.06(\mathrm{~d}, J=8.4 \mathrm{~Hz}, 1 \mathrm{H}), 6.90-6.83(\mathrm{~m}, 1 \mathrm{H}) ;{ }^{13} \mathrm{C} \mathrm{NMR}\left(126 \mathrm{MHz}, \mathrm{CDCl}_{3}\right) \delta$ 198.68, 163.16, $159.22\left({ }^{1} J_{\mathrm{C}-\mathrm{F}}=251.4 \mathrm{~Hz}\right), 137.18,133.50\left({ }^{4} J_{\mathrm{C}-\mathrm{F}}=2.3 \mathrm{~Hz}\right), 133.01$ $\left({ }^{3} J_{\mathrm{C}-\mathrm{F}}=8.2 \mathrm{~Hz}\right), 130.00\left({ }^{4} J_{\mathrm{C}-\mathrm{F}}=2.5 \mathrm{~Hz}\right), 126.46\left({ }^{2} J_{\mathrm{C}-\mathrm{F}}=15.1 \mathrm{~Hz}\right), 124.49\left({ }^{4} J_{\mathrm{C}-\mathrm{F}}=2.5\right.$ $\mathrm{Hz}), 119.88,119.18,116.43\left({ }^{2} J_{\mathrm{C}-\mathrm{F}}=21.4 \mathrm{~Hz}\right) ;{ }^{19} \mathrm{~F} \mathrm{NMR}\left(376 \mathrm{MHz}, \mathrm{CDCl}_{3}\right) \delta-112.35$ (m).<smiles>O=C(c1ccccc1O)c1ccccc1Cl</smiles>

(2-chlorophenyl)(2-hydroxyphenyl)methanone

(CAS: 70288-96-9) $\quad 2 c^{11}$

Following the standard procedure, the reaction of $\mathbf{1 c}$ afforded $\mathbf{2 c}$ as a white solid (20.3 $\mathrm{mg}, 87 \%)$ after flash chromatography: $\mathrm{TLC} \mathrm{R}_{\mathrm{f}}=0.60(\mathrm{PE} / \mathrm{EA}=20: 1) ;{ }^{1} \mathrm{H}$ NMR $(500$ 
$\left.\mathrm{MHz}, \mathrm{CDCl}_{3}\right) \delta 11.95(\mathrm{~s}, 1 \mathrm{H}), 7.55-7.43(\mathrm{~m}, 3 \mathrm{H}), 7.40(\mathrm{t}, J=7.3 \mathrm{~Hz}, 1 \mathrm{H}), 7.35(\mathrm{~d}, J$ $=6.8 \mathrm{~Hz}, 1 \mathrm{H}), 7.25(\mathrm{~d}, J=9.2 \mathrm{~Hz}, 1 \mathrm{H}), 7.07(\mathrm{~d}, J=8.4 \mathrm{~Hz}, 1 \mathrm{H}), 6.83(\mathrm{t}, J=7.6 \mathrm{~Hz}$, $1 \mathrm{H}) ;{ }^{13} \mathrm{C} \mathrm{NMR}\left(126 \mathrm{MHz}, \mathrm{CDCl}_{3}\right) \delta 200.72,163.35,137.51,137.32,133.64,131.37$, $130.99,130.22,128.69,126.87,119.53,119.25,118.51$.<smiles>O=C(c1ccccc1O)c1ccccc1Br</smiles>

(2-bromophenyl)(2-hydroxyphenyl)methanone (CAS:99515-47-6) 2d Following the standard procedure, the reaction of $\mathbf{1 d}$ afforded $\mathbf{2 d}$ as a white solid $(22.7 \mathrm{mg}, 82 \%)$ after flash chromatography: TLC $\mathrm{R}_{\mathrm{f}}=0.65(\mathrm{PE} / \mathrm{EA}=20: 1) ;{ }^{1} \mathrm{H}$ NMR $(500 \mathrm{MHz}$, $\left.\mathrm{CDCl}_{3}\right) \delta 11.93(\mathrm{~s}, 1 \mathrm{H}), 7.67(\mathrm{~d}, J=9.0 \mathrm{~Hz}, 1 \mathrm{H}), 7.52(\mathrm{ddd}, J=8.6,7.2,1.7 \mathrm{~Hz}, 1 \mathrm{H})$, $7.47-7.43(\mathrm{~m}, 1 \mathrm{H}), 7.38(\mathrm{td}, J=7.7,1.8 \mathrm{~Hz}, 1 \mathrm{H}), 7.33(\mathrm{dd}, J=7.4,1.8 \mathrm{~Hz}, 1 \mathrm{H}), 7.23$ $(\mathrm{dd}, J=8.0,1.7 \mathrm{~Hz}, 1 \mathrm{H}), 7.07(\mathrm{~d}, J=8.4,1.0 \mathrm{~Hz}, 1 \mathrm{H}), 6.84-6.81(\mathrm{~m}, 1 \mathrm{H}) ;{ }^{13} \mathrm{C} \mathrm{NMR}$ $\left(126 \mathrm{MHz} \mathrm{CDCl}_{3}\right) \delta 201.35,163.43,139.56,137.32,133.72,133.33,131.42,128.61$, $127.38,119.30,119.28,119.26,118.53$.<smiles>N#Cc1ccc(C(=O)c2ccccc2O)cc1</smiles>

4-(2-hydroxybenzoyl)benzonitrile $2 \mathrm{e}^{7}$ Following the standard procedure, the reaction of $\mathbf{1 e}$ afforded $\mathbf{2 e}$ as a yellow solid (16.3 $\mathrm{mg}, 73 \%)$ after flash chromatography: TLC R $\mathrm{R}_{\mathrm{f}}=0.65$ (PE / EA=10:1); ${ }^{1} \mathrm{H} \mathrm{NMR}\left(500 \mathrm{MHz}, \mathrm{CDCl}_{3}\right) \delta 11.77$ (s, $1 \mathrm{H}), 7.82(\mathrm{~d}, J=8.5 \mathrm{~Hz}, 2 \mathrm{H}), 7.76(\mathrm{~d}, J=8.5 \mathrm{~Hz}, 2 \mathrm{H}), 7.55(\mathrm{ddd}, J=8.6,7.3,1.6 \mathrm{~Hz}$, $1 \mathrm{H}), 7.45(\mathrm{~d}, J=8.0,1.5 \mathrm{H}, 1 \mathrm{H}), 7.10(\mathrm{~d}, J=8.4 \mathrm{~Hz}, 1 \mathrm{H}), 6.93-6.88(\mathrm{~m}, 1 \mathrm{H}) ;{ }^{13} \mathrm{C}$ NMR $\left(126 \mathrm{MHz}, \mathrm{CDCl}_{3}\right) \delta 199.87,163.56,141.70,137.35,133.16,132.35,129.57$, 119.20, 118.94, 118.64, 117.99, 115.48 . 


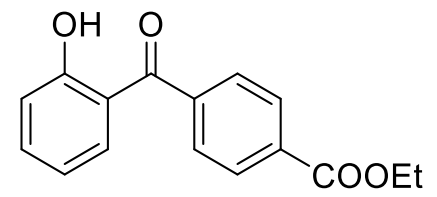

ethyl 4-(2-hydroxybenzoyl)benzoate (CAS: 1228435-75-3) $2 \mathrm{f}^{7}$ Following the standard procedure, the reaction of $\mathbf{1 f}$ afforded $\mathbf{2 f}$ as a yellow solid (20.8 $\mathrm{mg}, 77 \%$ ) after flash chromatography: TLC $\mathrm{R}_{\mathrm{f}}=0.60(\mathrm{PE} / \mathrm{EA}=10: 1) ;{ }^{1} \mathrm{H} \mathrm{NMR}(500 \mathrm{MHz}$, $\left.\mathrm{CDCl}_{3}\right) \delta 11.93(\mathrm{~s}, 1 \mathrm{H}), 8.18(\mathrm{~d}, J=8.5 \mathrm{~Hz}, 2 \mathrm{H}), 7.72(\mathrm{~d}, J=8.5 \mathrm{~Hz}, 2 \mathrm{H}), 7.55-7.50$ (m, 2H), $7.08(\mathrm{~d}, J=8.4 \mathrm{~Hz}, 1 \mathrm{H}), 6.89-6.86(\mathrm{~m}, 1 \mathrm{H}), 4.43(\mathrm{q}, J=7.1 \mathrm{~Hz}, 2 \mathrm{H}), 1.43$ (t, $J=7.1 \mathrm{~Hz}, 3 \mathrm{H}) ;{ }^{13} \mathrm{C} \mathrm{NMR}\left(126 \mathrm{MHz}, \mathrm{CDCl}_{3}\right) \delta 200.95,165.68,163.32,141.51,136.79$, $133.35,133.23,129.50,128.86,118.89,118.84,118.57,61.47,14.29$.<smiles>O=Cc1ccc(C(=O)c2ccccc2O)cc1</smiles>

4-(2-hydroxybenzoyl)benzaldehyde (CAS: 1228435-75-3) $\mathbf{2 g}^{12}$ Following the standard procedure, the reaction of $\mathbf{1 g}$ afforded $\mathbf{2 g}$ as a white solid (17.1 $\mathrm{mg}, 76 \%)$ after flash chromatography: TLC $\mathrm{R}_{\mathrm{f}}=0.40(\mathrm{PE} / \mathrm{EA}=10: 1) ;{ }^{1} \mathrm{H} \mathrm{NMR}(500 \mathrm{MHz}$, $\left.\mathrm{CDCl}_{3}\right) \delta 11.88(\mathrm{~s}, 1 \mathrm{H}), 10.13(\mathrm{~s}, 1 \mathrm{H}), 8.02(\mathrm{~d}, J=8.3 \mathrm{~Hz}, 2 \mathrm{H}), 7.81(\mathrm{~d}, J=8.1 \mathrm{~Hz}, 2 \mathrm{H})$, 7.56-7.52 (m, 1H), 7.49 (dd, $J=8.0,1.6 \mathrm{~Hz}, 1 \mathrm{H}), 7.09$ (d, $J=8.0 \mathrm{~Hz}, 1 \mathrm{H}), 6.90-6.87$ (m, $1 \mathrm{H}) ;{ }^{13} \mathrm{C} \mathrm{NMR}\left(126 \mathrm{MHz}, \mathrm{CDCl}_{3}\right) \delta 200.74,191.55,163.52,143.00,138.28,137.14$, $133.39,129.67,129.60,119.09,118.91,118.81$.

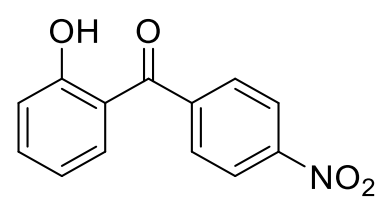

(2-hydroxyphenyl)(4-nitrophenyl)methanone

(CAS: $\quad 68223-20-1) \quad 2 h^{7}$

Following the standard procedure, irradiation for $24 \mathrm{~h}$, the reaction of $\mathbf{1 h}$ afforded $\mathbf{2 h}$ as a yellow solid (17.3 mg, 71\%) after flash chromatography: TLC $\mathrm{R}_{\mathrm{f}}=0.50$ (PE / EA $=10: 1) ;{ }^{1} \mathrm{H}$ NMR $\left(500 \mathrm{MHz}, \mathrm{CDCl}_{3}\right) \delta 11.77(\mathrm{~s}, 1 \mathrm{H}), 8.37(\mathrm{~d}, J=8.8 \mathrm{~Hz}, 2 \mathrm{H}), 7.83(\mathrm{~d}$, $J=8.8 \mathrm{~Hz}, 2 \mathrm{H}), 7.57(\mathrm{ddd}, J=8.7,7.3,1.6 \mathrm{~Hz}, 1 \mathrm{H}), 7.45(\mathrm{dd}, J=8.0,1.6 \mathrm{~Hz}, 1 \mathrm{H}), 7.11$ 
$(\mathrm{d}, J=8.4 \mathrm{~Hz}, 1 \mathrm{H}), 6.94-6.88(\mathrm{~m}, 1 \mathrm{H}) ;{ }^{13} \mathrm{C} \mathrm{NMR}\left(126 \mathrm{MHz}, \mathrm{CDCl}_{3}\right) \delta$ 199.71, 163.61, $149.67,143.36,137.50,133.17,130.01,123.78,119.28,119.00,118.70$.<smiles>COc1cccc(OC)c1C(=O)c1ccccc1O</smiles>

(2, 6-dimethoxyphenyl)(2-hydroxyphenyl)methanone (CAS: 129103-86-2) $2 \mathbf{i}^{13}$ Following the standard procedure, the reaction of $\mathbf{1} \mathbf{i}$ afforded $\mathbf{2} \mathbf{i}$ as a white solid (19.6 $\mathrm{mg}, 76 \%)$. after flash chromatography: TLC $\mathrm{R}_{\mathrm{f}}=0.55$ (PE / EA = 10:1); ${ }^{1} \mathrm{H}$ NMR (500 $\left.\mathrm{MHz}, \mathrm{CDCl}_{3}\right) \delta 12.15(\mathrm{~s}, 1 \mathrm{H}), 7.45(\mathrm{ddd}, J=8.7,7.2,1.7 \mathrm{~Hz}, 1 \mathrm{H}), 7.37(\mathrm{t}, J=8.4 \mathrm{~Hz}$, $1 \mathrm{H}), 7.28(\mathrm{dd}, J=8.0,1.7 \mathrm{~Hz}, 1 \mathrm{H}), 7.02(\mathrm{dd}, J=8.4,1.1 \mathrm{~Hz}, 1 \mathrm{H}), 6.78(\mathrm{ddd}, J=8.1,7.1$, $1.1 \mathrm{~Hz}, 1 \mathrm{H}), 6.63(\mathrm{~d}, J=8.4 \mathrm{~Hz}, 2 \mathrm{H}), 3.73(\mathrm{~s}, 6 \mathrm{H}) ;{ }^{13} \mathrm{C} \mathrm{NMR}\left(126 \mathrm{MHz}, \mathrm{CDCl}_{3}\right) \delta$ 201.26, 162.53, 157.31, 136.36, 133.02, 131.30, 121.00, 118.87, 117.86, 116.47, 104.01, 55.91.<smiles>CCCc1ccccc1C(=O)c1c(O)cccc1C(C)C</smiles>

(2,6-diisopropylphenyl)(2-hydroxyphenyl)methanone $2 \mathbf{j}$ Following the standard procedure, the reaction of $\mathbf{1} \mathbf{j}$ afforded $\mathbf{2} \mathbf{j}$ as a white solid $(21.7 \mathrm{mg}, 77 \%)$ after flash chromatography: TLC $\mathrm{R}_{\mathrm{f}}=0.40(\mathrm{PE} / \mathrm{EA}=100: 1) ;{ }^{1} \mathrm{H} \mathrm{NMR}\left(500 \mathrm{MHz}, \mathrm{CDCl}_{3}\right) \delta$ $12.30(\mathrm{~s}, 1 \mathrm{H}), 7.40(\mathrm{t}, J=7.8 \mathrm{~Hz}, 1 \mathrm{H})), 7.40(\mathrm{t}, J=7.8 \mathrm{~Hz}, 1 \mathrm{H}), 7.16(\mathrm{~d}, J=7.8 \mathrm{~Hz}, 2 \mathrm{H})$, $7.10(\mathrm{~d}, J=8.0 \mathrm{~Hz}, 1 \mathrm{H}), 6.98(\mathrm{~d}, J=8.4 \mathrm{~Hz}, 1 \mathrm{H}), 6.69$ (t, $J=7.6 \mathrm{~Hz}, 1 \mathrm{H}), 2.58$ (hepta, $6.4 \mathrm{~Hz}, 2 \mathrm{H}) ; 1.11(\mathrm{~d}, J=6.8 \mathrm{~Hz}, 6 \mathrm{H}), 1.01(\mathrm{~d}, J=6.8 \mathrm{~Hz}, 6 \mathrm{H}) .{ }^{13} \mathrm{C} \mathrm{NMR}(126 \mathrm{MHz}$, $\left.\mathrm{CDCl}_{3}\right) \delta 207.10,162.61,145.10,137.08,135.92,133.12,129.90,123.21,121.43$, 119.10, 118.40, 31.34, 25.06, 23.44; IR (neat): 3447, 3064, 2963, 2926, 2869, 1625, 1576, 1481, 1447, 1340, 1302, 1251, 1219, 1144, 926, 805, 766, $618 \mathrm{~cm}^{-1}$; HRMS-ESI (m/z): [M-H] ${ }^{-}$calc'd for $\mathrm{C}_{19} \mathrm{H}_{21} \mathrm{O}_{2}$ 281.1547, found 281.1547.

\section{4-isopropyl-9H-xanthen-9-one 5}

the reaction $\mathbf{1 j}$ afforded $\mathbf{5}$ as a white solid (3.9 $\mathrm{mg}, 16 \%$ ) after flash chromatography 
TLC $\mathrm{R}_{\mathrm{f}}=0.40(\mathrm{PE} / \mathrm{EA}=30: 1) ;{ }^{1} \mathrm{H}$ NMR $(500 \mathrm{MHz}$, Chloroform- $d$ ) $\delta 8.35(\mathrm{dd}, J=$ 7.9, $1.6 \mathrm{~Hz}, 1 \mathrm{H}), 8.21$ (dd, $J=7.9,1.6 \mathrm{~Hz}, 1 \mathrm{H}), 7.74(\mathrm{ddd}, J=8.5,7.1,1.7 \mathrm{~Hz}, 1 \mathrm{H})$, $7.65(\mathrm{dd}, J=7.4,1.5 \mathrm{~Hz}, 1 \mathrm{H}), 7.54$ (d, $J=8.4 \mathrm{~Hz}, 1 \mathrm{H}), 7.39$ (t, $J=7.5 \mathrm{~Hz}, 1 \mathrm{H}), 7.35$ (t, $J=7.7 \mathrm{~Hz}, 1 \mathrm{H}), 3.72$ (hept, $J=6.9 \mathrm{~Hz}, 1 \mathrm{H}), 1.39$ (d, $J=6.9 \mathrm{~Hz}, 6 \mathrm{H}) .{ }^{13} \mathrm{C} \mathrm{NMR}$ $\left(126 \mathrm{MHz} \mathrm{CDCl}_{3}\right) \delta 177.82,156.14,153.72,137.64,134.82,131.69,126.82,124.33$, 123.98, 123.84, 121.91, 121.70, 118.14, 27.23, 22.89; IR (neat): 2960, 2870, 1666, 1616, 1597, 1483, 1360, 1334, 1226, 1201, 1147, 1092, 905, 754, $688 \mathrm{~cm}^{-1}$; HRMS-ESI (m/z): $[\mathrm{M}+\mathrm{H}]^{+}$calc'd for $\mathrm{C}_{16} \mathrm{H}_{15} \mathrm{O}_{2}$ 239.1067, found 239.1066.<smiles>Cc1ccc(C(=O)c2ccccc2O)c(C)c1</smiles>

(2,4-dimethylphenyl)(2-hydroxyphenyl)methanone (CAS: $\quad$ 143824-87-7) $\quad \mathbf{2 k}^{14}$ Following the standard procedure, the reaction of $\mathbf{1 k}$ afforded $\mathbf{2 k}$ as a white solid (13.2 mg, 58\%) after flash chromatography: TLC $\mathrm{R}_{\mathrm{f}}=0.40(\mathrm{PE} / \mathrm{EA}=100: 1) ;{ }^{1} \mathrm{H}$ $\operatorname{NMR}\left(500 \mathrm{MHz}, \mathrm{CDCl}_{3}\right) \delta 12.30(\mathrm{~s}, 1 \mathrm{H}), 7.49(\mathrm{t}, J=7.8 \mathrm{~Hz}, 1 \mathrm{H}), 7.37-7.31(\mathrm{~m}, 1 \mathrm{H})$, $7.18(\mathrm{~d}, J=7.7 \mathrm{~Hz}, 1 \mathrm{H}), 7.12(\mathrm{~s}, 1 \mathrm{H}), 7.07(\mathrm{dd}, J=16.1,8.1 \mathrm{~Hz}, 2 \mathrm{H}), 6.81$ (t, $J=7.6 \mathrm{~Hz}$, 1H), 2.39 (s, 3H), 2.29 (s, 3H). ${ }^{13} \mathrm{C} \mathrm{NMR}\left(126 \mathrm{MHz}, \mathrm{CDCl}_{3}\right) \delta 204.57,163.36,140.55$, 136.73, 135.93, 135.11, 133.90, 131.84, 128.06, 126.04, 120.18, 118.90, 118.38, 21.51, 19.75 .

2,4-dimethyl-9H-xanthen-9-one (CAS: 40305-53-1) $6^{15}$ the reaction $2 \mathrm{k}$ afforded 6 as a white solid (5.0 mg, 22\%) after flash chromatography TLC $\mathrm{R}_{\mathrm{f}}=0.40(\mathrm{PE} / \mathrm{EA}$ $=30: 1) ;{ }^{1} \mathrm{H}$ NMR $\left(500 \mathrm{MHz}, \mathrm{CDCl}_{3}\right) \delta 8.34(\mathrm{dd}, J=8.0,1.6 \mathrm{~Hz}, 1 \mathrm{H}), 7.97(\mathrm{~s}, 1 \mathrm{H})$, $7.75-7.69(\mathrm{~m}, 1 \mathrm{H}), 7.52(\mathrm{~d}, J=8.4 \mathrm{~Hz}, 1 \mathrm{H}), 7.37(\mathrm{dd}, J=14.0,6.7 \mathrm{~Hz}, 2 \mathrm{H}), 2.53$ (s, 3H), $2.43(\mathrm{~s}, 3 \mathrm{H}) ;{ }^{13} \mathrm{C} \mathrm{NMR}\left(126 \mathrm{MHz}, \mathrm{CDCl}_{3}\right) \delta 177.79,156.20,152.94,137.24$, 134.63, 133.21, 127.08, 126.82, 123.79, 123.73, 121.74, 121.45, 118.16, 20.96, 15.90 . 
<smiles>COc1ccc2ccccc2c1C(=O)c1ccccc1O</smiles>

(2-hydroxyphenyl)(2-methoxynaphthalen-1-yl)methanone 21 Following the standard procedure, the reaction of $\mathbf{2 l}$ afforded $\mathbf{1 1}$ as a white solid (15.7 $\mathrm{mg}, 56 \%)$ after flash chromatography: TLC $\mathrm{R}_{\mathrm{f}}=0.50(\mathrm{PE} / \mathrm{EA}=50: 1) ;{ }^{1} \mathrm{H} \mathrm{NMR}(500 \mathrm{MHz}$, $\left.\mathrm{CDCl}_{3}\right) \delta 12.34(\mathrm{~s}, 1 \mathrm{H}), 7.99(\mathrm{~d}, J=9.1 \mathrm{~Hz}, 1 \mathrm{H}), 7.85(\mathrm{~d}, J=7.8 \mathrm{~Hz}, 1 \mathrm{H}), 7.52-7.45$ (m, 2H), $7.43-7.34(\mathrm{~m}, 3 \mathrm{H}), 7.18(\mathrm{dd}, J=8.0,1.6 \mathrm{~Hz}, 1 \mathrm{H}), 7.09(\mathrm{~d}, J=8.4 \mathrm{~Hz}, 1 \mathrm{H})$, $6.75-6.68(\mathrm{~m}, 1 \mathrm{H}), 3.87(\mathrm{~s}, 3 \mathrm{H}) ;{ }^{13} \mathrm{C} \mathrm{NMR}\left(126 \mathrm{MHz}, \mathrm{CDCl}_{3}\right) \delta$ 203.72, 162.92, $153.87,136.96,133.49,131.74,131.53,128.79,128.29,127.78,124.37,123.99$, 121.48, 121.27, 119.23, 118.26, 113.07, 56.71; IR (neat): 3447, 3061, 2939, 2842, 1626, $1594,1510,1482,1270,1253,1239,1214,1148,1078,902,817,806,757,635 \mathrm{~cm}-1$; HRMS-ESI (m/z): [M+2Na-H] $]^{+}$calc'd for $\mathrm{C}_{18} \mathrm{H}_{15} \mathrm{O}_{3}$ 301.0840, found 301.0835.<smiles>O=C(c1ccccc1O)c1cccc2cccnc12</smiles>

(2-hydroxyphenyl)(quinolin-8-yl)methanone (CAS: $\quad$ 1101859-05-5) $\quad \mathbf{2 m}^{16}$ Following the standard procedure, Acetone / HFIP $=1: 1$ as solvent $(0.05 \mathrm{M})$, the reaction of $\mathbf{1 m}$ afforded $\mathbf{2 m}$ as a pale solid $(6.6 \mathrm{mg}, 26 \%)$ after flash chromatography: TLC $\mathrm{R}_{\mathrm{f}}=0.50(\mathrm{PE} / \mathrm{EA}=2: 1) ;{ }^{1} \mathrm{H} \mathrm{NMR}\left(500 \mathrm{MHz}, \mathrm{CDCl}_{3}\right) \delta 12.29(\mathrm{~s}, 1 \mathrm{H}), 8.89(\mathrm{dd}$, $J=4.2,1.7 \mathrm{~Hz}, 1 \mathrm{H}), 8.24(\mathrm{dd}, J=8.3,1.7 \mathrm{~Hz}, 1 \mathrm{H}), 7.99(\mathrm{dd}, J=8.2,1.4 \mathrm{~Hz}, 1 \mathrm{H})$, $7.72(\mathrm{dd}, J=7.0,1.4 \mathrm{~Hz}, 1 \mathrm{H}), 7.65(\mathrm{dd}, J=8.1,7.1 \mathrm{~Hz}, 1 \mathrm{H}), 7.50-7.43(\mathrm{~m}, 2 \mathrm{H})$, $7.14(\mathrm{dd}, J=8.0,1.7 \mathrm{~Hz}, 1 \mathrm{H}), 7.08(\mathrm{~d}, J=8.4 \mathrm{~Hz}, 1 \mathrm{H}), 6.74-6.68(\mathrm{~m}, 1 \mathrm{H}) .{ }^{13} \mathrm{C}$ NMR $\left(126 \mathrm{MHz}, \mathrm{CDCl}_{3}\right) \delta 203.74,163.14,151.31,145.87,137.86,136.84,136.25$, $134.15,130.13,128.36,128.26,125.93,122.00,120.91,118.88,118.39$.<smiles>CCOC(=O)c1ccc(C(=O)c2cc(C)ccc2O)cc1</smiles> 
ethyl 4-(2-hydroxy-5-methylbenzoyl)benzoate 2n Following the standard procedure, the reaction of $\mathbf{1 n}$ afforded $\mathbf{2 n}$ as a white solid (18.8 $\mathrm{mg}, 66 \%)$, after flash chromatography: TLC $\mathrm{R}_{\mathrm{f}}=0.60(\mathrm{PE} / \mathrm{EA}=20: 1) ;{ }^{1} \mathrm{H}$ NMR $\left(500 \mathrm{MHz}, \mathrm{CDCl}_{3}\right) \delta 11.75$ (s, 1H), $8.18(\mathrm{~d}, J=8.1 \mathrm{~Hz}, 2 \mathrm{H}), 7.71(\mathrm{~d}, J=8.1 \mathrm{~Hz}, 2 \mathrm{H}), 7.35(\mathrm{~d}, J=8.5 \mathrm{~Hz}, 1 \mathrm{H}), 7.26$ (s, 1H), 6.99 (d, $J=8.5 \mathrm{~Hz}, 1 \mathrm{H}), 4.44$ (q, $J=7.1 \mathrm{~Hz}, 2 \mathrm{H}), 2.24$ (s, 3H), 1.43 (t, $J=7.1$ $\mathrm{Hz}, 3 \mathrm{H}) ;{ }^{13} \mathrm{C} \mathrm{NMR}\left(126 \mathrm{MHz}, \mathrm{CDCl}_{3}\right) \delta 201.07,165.89,161.43,141.87,133.27$, 133.08, 129.64, 128.94, 128.19, 118.73, 118.49, 61.61, 20.61, 14.46; IR (neat): 2981, 1721, 1632, 1608, 1503, 1482, 1367, 1336, 1275, 1244, 1222, 1137, 1124, 1104, 957, 846, 828, 786, 762, 710, $670 \mathrm{~cm}^{-1}$; HRMS-ESI $(\mathrm{m} / \mathrm{z}):[\mathrm{M}+\mathrm{H}]^{+}$calc'd for $\mathrm{C}_{17} \mathrm{H}_{17} \mathrm{O}_{4}$ 285.1121, found 285.1118 .<smiles>CC(=O)c1cc(-c2ccccc2)ccc1O</smiles>

(4-hydroxy-[1,1'-biphenyl]-3-yl)(mesityl)methanone 20 Following the standard procedure, the reaction of $\mathbf{1 0}$ afforded $\mathbf{2 0}$ as a white solid $(23.8 \mathrm{mg}, 75 \%)$, after flash chromatography: TLC $\mathrm{R}_{\mathrm{f}}=0.30(\mathrm{PE} / \mathrm{EA}=100: 1) ;{ }^{1} \mathrm{H} \mathrm{NMR}\left(500 \mathrm{MHz}, \mathrm{CDCl}_{3}\right) \delta$ $12.40(\mathrm{~s}, 1 \mathrm{H}), 7.74(\mathrm{dd}, J=8.6,2.4 \mathrm{~Hz}, 1 \mathrm{H}), 7.41-7.34(\mathrm{~m}, 5 \mathrm{H}), 7.33-7.27(\mathrm{~m}, 1 \mathrm{H})$, $7.15(\mathrm{~d}, J=8.6 \mathrm{~Hz}, 1 \mathrm{H}), 6.93(\mathrm{~s}, 2 \mathrm{H}), 2.35$ (s, 3H), 2.17 (s, 6H); ${ }^{13} \mathrm{C}$ NMR (126 MHz, $\left.\mathrm{CDCl}_{3}\right) \delta 206.94,162.36,139.74,139.08,135.81,135.23,133.92,132.69,130.75$, 128.80, 128.48, 127.10, 126.67, 120.38, 118.80, 21.19, 19.39; IR (neat): 3030, 2912, $1631,1612,1473,1335,1277,1236,1221,1171,1108,961,930,835,803,770,754$, $696 \mathrm{~cm}^{-1}$; HRMS-ESI (m/z): [M-H] $]^{-}$calc'd for $\mathrm{C}_{22} \mathrm{H}_{19} \mathrm{O}_{2}$ 315.1391, found 315.1387.<smiles>CC(=O)c1cc(C#CCBr)ccc1O</smiles>

(5-(3,3-dimethylbut-1-yn-1-yl)-2-hydroxyphenyl)(mesityl)methanone

Following the standard procedure, the reaction of $\mathbf{1 p}$ afforded $\mathbf{2 p}$ as a white solid (24.1 mg, 75\%), after flash chromatography: TLC $\mathrm{R}_{\mathrm{f}}=0.30$ (PE / EA=100:1); ${ }^{1} \mathrm{H}$ NMR 
$\left(500 \mathrm{MHz}, \mathrm{CDCl}_{3}\right) \delta 12.48(\mathrm{~s}, 1 \mathrm{H}), 7.50(\mathrm{dd}, J=8.6,2.0 \mathrm{~Hz}, 1 \mathrm{H}), 7.20(\mathrm{~d}, J=2.0 \mathrm{~Hz}$, $1 \mathrm{H}), 6.97(\mathrm{~d}, J=8.6 \mathrm{~Hz}, 1 \mathrm{H}), 6.93(\mathrm{~s}, 2 \mathrm{H}), 2.36(\mathrm{~s}, 3 \mathrm{H}), 2.12(\mathrm{~s}, 6 \mathrm{H}), 1.26(\mathrm{~s}, 9 \mathrm{H}) ;{ }^{13} \mathrm{C}$ NMR $\left(126 \mathrm{MHz} \mathrm{CDCl}_{3}\right) \delta 206.74,162.44,140.55,139.21,135.32,135.09,134.16$, 128.61, 120.17, 118.65, 115.45, 97.48, 77.83, 31.12, 27.98, 21.35, 19.49; IR (neat): 2970, 2924 2867, 2220, 1629, 1612, 1587, 1481, 1471, 1330, 1293, 1238, 1195, 1165 , 841, 800, $789 \mathrm{~cm}^{-1}$; HRMS-ESI (m/z): [M-H] $]^{-}$calc'd for $\mathrm{C}_{22} \mathrm{H}_{23} \mathrm{O}_{2}$ 319.1704, found 319.1700 .<smiles>COc1ccc(C(C)=O)c(O)c1</smiles>

(2-hydroxy-4-methoxyphenyl)(mesityl)methanone $2 q$ Following the standard procedure, the reaction of $\mathbf{1 q}$ afforded $\mathbf{2 q}$ as a white solid $(23.1 \mathrm{mg}, 86 \%)$ after flash chromatography: TLC $\mathrm{R}_{\mathrm{f}}=0.50(\mathrm{PE} / \mathrm{EA}=30: 1) ;{ }^{1} \mathrm{H} \mathrm{NMR}\left(500 \mathrm{MHz}, \mathrm{CDCl}_{3}\right) \delta$ $12.84(\mathrm{~s}, 1 \mathrm{H}), 7.06(\mathrm{~d}, J=8.9,1.3 \mathrm{~Hz}, 1 \mathrm{H}), 6.90(\mathrm{~s}, 2 \mathrm{H}), 6.49$ (s, 1H), 6.32 (d, J = 8.9 $\mathrm{Hz}, 1 \mathrm{H}), 3.84$ (s, 3H), 2.33 (s, 3H), 2.12 (s, 6H); $\left.{ }^{13} \mathrm{C} \mathrm{NMR} \mathrm{(126} \mathrm{MHz,} \mathrm{CDCl}_{3}\right) \delta 204.56$, 166.73, 165.95, 138.87, 135.77, 134.51, 134.07, 128.41, 114.72, 108.14, 100.99, 55.76, 21.28, 19.38; IR (neat): 3450, 2919, 2847, 1613, 1505, 1441, 1377, 1341, 1278, 1259, 1207, 1103, 1027, 904, 843, 798, $610 \mathrm{~cm}^{-1}$; HRMS-ESI $(\mathrm{m} / \mathrm{z}):[\mathrm{M}+\mathrm{H}]^{+}$calc'd for $\mathrm{C}_{17} \mathrm{H}_{19} \mathrm{O}_{3} 271.1329$, found 271.1328 .<smiles>CC(=O)c1ccc(Cl)cc1O</smiles>

(2-hydroxy-4-methoxyphenyl)(mesityl)methanone $2 r$ Following the standard procedure, the reaction of $\mathbf{1 r}$ afforded $\mathbf{2 r}$ as a white solid $(22.4 \mathrm{mg}, 81 \%)$ after flash chromatography: TLC $\mathrm{R}_{\mathrm{f}}=0.25(\mathrm{PE} / \mathrm{EA}=100: 1) ;{ }^{1} \mathrm{H} \mathrm{NMR}\left(500 \mathrm{MHz}, \mathrm{CDCl}_{3}\right) \delta$ $12.48(\mathrm{~s}, 1 \mathrm{H}), 7.11(\mathrm{~d}, J=8.5 \mathrm{~Hz}, 1 \mathrm{H}), 7.07(\mathrm{~d}, J=2.0 \mathrm{~Hz}, 1 \mathrm{H}), 6.92(\mathrm{~s}, 2 \mathrm{H}), 6.76(\mathrm{dd}$, $J=8.5,2.0 \mathrm{~Hz}, 1 \mathrm{H}), 2.34(\mathrm{~s}, 3 \mathrm{H}), 2.11(\mathrm{~s}, 6 \mathrm{H}) .{ }^{13} \mathrm{C} \mathrm{NMR}\left(126 \mathrm{MHz}, \mathrm{CDCl}_{3}\right) \delta 206.12$, $163.60,142.87,139.42,135.15,134.07,133.86,128.59,120.14,119.05,118.59,21.30$, 
19.39. IR (neat): $3452,2955,2917,2858,1622,1564,1482,1327,1285,1233,1217$, 1178, 1076, 930, 881, 788, $510 \mathrm{~cm}^{-1}$; HRMS-ESI (m/z): [M-H] calc'd for $\mathrm{C}_{16} \mathrm{H}_{14} \mathrm{O}_{2} \mathrm{Cl}$ 273.0688, found 273.0685 .<smiles>CC(=O)c1ccc(Br)cc1O</smiles>

(4-bromo-2-hydroxyphenyl)(mesityl)methanone $2 s$ Following the standard procedure, the reaction of $\mathbf{1 s}$ afforded $2 \mathrm{~s}$ as a white solid $(25.7 \mathrm{mg}, 80 \%)$ after flash chromatography: TLC $\mathrm{R}_{\mathrm{f}}=0.25(\mathrm{PE} / \mathrm{EA}=100: 1) ;{ }^{1} \mathrm{H}$ NMR $\left(500 \mathrm{MHz}, \mathrm{CDCl}_{3}\right) \delta$ $12.43(\mathrm{~s}, 1 \mathrm{H}), 7.26(\mathrm{~d}, J=2.0 \mathrm{~Hz}, 1 \mathrm{H}), 7.03(\mathrm{~d}, J=8.5 \mathrm{~Hz}, 1 \mathrm{H}), 6.94-6.89$ (m, 3H), $2.34(\mathrm{~s}, 3 \mathrm{H}), 2.11(\mathrm{~s}, 6 \mathrm{H}) ;{ }^{13} \mathrm{C} \mathrm{NMR}\left(126 \mathrm{MHz}, \mathrm{CDCl}_{3}\right) \delta$ 206.37, 163.37, 139.44, $135.12,134.09,133.80,131.59,128.60,123.01,121.73,119.35,21.32,19.40$; IR (neat): 3438, 2944, 2914, 2855, 1624, 1611, 1563, 1477, 1324, 1283, 1230, 1218, 1177, 1068, 959, 921, 867, $787 \mathrm{~cm}^{-1}$; HRMS-ESI $(\mathrm{m} / \mathrm{z}):[\mathrm{M}+\mathrm{H}]+$ calc'd for $\mathrm{C}_{16} \mathrm{H}_{16} \mathrm{BrO}_{2} 319.0328$, found 319.0322 .<smiles>CC(=O)c1ccc(C#N)cc1O</smiles>

3-hydroxy-4-(2,4,6-trimethylbenzoyl)benzonitrile $2 \mathrm{t}$ Following the standard procedure, the reaction of $\mathbf{1 t}$ afforded $\mathbf{2 t}$ as a white solid (15.0 $\mathrm{mg}, 57 \%)$, after flash chromatography: TLC $\mathrm{R}_{\mathrm{f}}=0.45(\mathrm{PE} / \mathrm{EA}=20: 1) ;{ }^{1} \mathrm{H} \mathrm{NMR}\left(500 \mathrm{MHz}, \mathrm{CDCl}_{3}\right) \delta$ 12.35 (s, 1H), 7.35 (d, $J=1.4 \mathrm{~Hz}, 1 \mathrm{H}), 7.29$ (d, 8.2 Hz 1H), 7.04 (dd, $J=8.2,1.5 \mathrm{~Hz}$, 1H), 6.93 (s, 2H), 2.35 (s, 3H), $\left.2.09(\mathrm{~s}, 6 \mathrm{H}) .{ }^{13} \mathrm{C} \mathrm{NMR} \mathrm{(126} \mathrm{MHz,} \mathrm{CDCl}_{3}\right) \delta 206.84$, $162.44,139.95,134.46,134.11,133.49,128.76,122.86,122.66,122.24,119.39$, 117.57, 21.32, 19.44; IR (neat): 2921, 2233, 1632, 1613, 1561, 1492, 1410, 1345, 1325 , 1294, 1247, 1222, 1179, 1149, 972, 896, 852, 791, 747, $669 \mathrm{~cm}^{-1}$; HRMS-ESI (m/z): $[\mathrm{M}+\mathrm{H}]^{+}$calc'd for $\mathrm{C}_{17} \mathrm{H}_{14} \mathrm{NO}_{2} 264.1039$, found 264.1030. 
<smiles>CC(=O)c1cc(C(F)(F)F)ccc1O</smiles>

(2-hydroxy-4-(trifluoromethyl)phenyl)(mesityl)methanone $2 u$ Following the standard procedure, the reaction of $\mathbf{1 u}$ afforded $\mathbf{2 u}$ as a white solid (26.3 $\mathrm{mg}, 85 \%)$, after flash chromatography: TLC $\mathrm{R}_{\mathrm{f}}=0.30(\mathrm{PE} / \mathrm{EA}=100: 1) ;{ }^{1} \mathrm{H} \mathrm{NMR}(500 \mathrm{MHz}$, $\left.\mathrm{CDCl}_{3}\right) \delta 12.69(\mathrm{~s}, 1 \mathrm{H}), 7.71(\mathrm{dd}, J=8.8,2.2 \mathrm{~Hz}, 1 \mathrm{H}), 7.45(\mathrm{~d}, J=1.6 \mathrm{~Hz}, 1 \mathrm{H}), 7.16(\mathrm{~d}$, $J=8.8 \mathrm{~Hz}, 1 \mathrm{H}), 6.94(\mathrm{~s}, 2 \mathrm{H}), 2.36(\mathrm{~s}, 3 \mathrm{H}), 2.12(\mathrm{~s}, 6 \mathrm{H}) ;{ }^{13} \mathrm{C} \mathrm{NMR}\left(126 \mathrm{MHz}, \mathrm{CDCl}_{3}\right) \delta$ $206.63,165.34,139.84,134.40,134.11,133.31\left({ }^{3} J_{\mathrm{C}-\mathrm{F}}=3.3 \mathrm{~Hz}\right), 130.15\left({ }^{3} J_{\mathrm{C}-\mathrm{F}}=3.9 \mathrm{~Hz}\right)$, $128.84,123.75\left({ }^{1} J_{\mathrm{C}-\mathrm{F}}=272.1 \mathrm{~Hz}\right), 121.91\left({ }^{2} J_{\mathrm{C}-\mathrm{F}}=33.5 \mathrm{~Hz}\right), 119.88,119.47,21.33$, 19.47; ${ }^{19} \mathrm{~F}$ NMR (376 MHz, $\left.\mathrm{CDCl}_{3}\right) \delta$-62.16 (m); IR (neat): 2928, 1638, 1596, 1490, 1359, 1320, 1292, 1244, 1225, 1174, 1124, 1074, $839 \mathrm{~cm}^{-1}$; HRMS-ESI (m/z): $[\mathrm{M}+\mathrm{H}]^{+}$ calc'd for $\mathrm{C}_{17} \mathrm{H}_{14} \mathrm{~F}_{3} \mathrm{O}_{2}$ 307.0951, found 307.0959.

\section{Gram Scale Synthesis of $1 \mathbf{r}$}

Ketoacid 1r (1.75 g, $5.5 \mathrm{mmol})$, Acr-Mes- $\mathrm{ClO}_{4}$ (45.3 mg, $0.11 \mathrm{mmol}, 0.02$ eq.), and BIOAc (336 mg, $1.1 \mathrm{mmol}, 0.2$ eq.) were placed in a $100 \mathrm{~mL}$ schlenk tube vial equipped with a magnetic stir bar. After $55 \mathrm{~mL}$ Acetone (treated by $4 \AA$ MS) was added into the tube via a syringe, the reaction mixture was degassed using a freeze-pump-thaw procedure (3 times) and exposed to blue LEDs (80 W LED light bulbs $10 \mathrm{~cm}$ away from the vial at room temperature with stirring for $12 \mathrm{~h}$ ), the reaction mixture was concentrated and purified directly by column chromatography to afford $2 \mathbf{r}$ as a white solid $(1.21 \mathrm{~g}, 80 \%)$. 


\section{References}

1. Jia, K.; Li, J.; Chen, Y., Chem. Eur. J. 2018, 24, 3174-3177.

2. Vogler, T.; Studer, A., Synthesis 2008, 2008, 1979-1993.

3. Cismesia, M. A.; Yoon, T. P., Chem. Sci. 2015, 6, 5426-5434.

4. Parker, C. A.; Hatchard, C. G.; Bowen Edmund, J., Proc. Roy. Soc. (London) A. 1962, 269, 574-584.

5. Liu, Z.; Tan, H.; Wang, L.; Fu, T.; Xia, Y.; Zhang, Y.; Wang, J., Angew. Chem. Int. Ed. 2015, 54, 3056-3060.

6. Wadhwa, K.; Yang, C.; West, P. R.; Deming, K. C.; Chemburkar, S. R.; Reddy, R. E., Synthetic Commun. 2008, 38, 4434-4444.

7. Janssen-Muller, D.; Singha, S.; Lied, F.; Gottschalk, K.; Glorius, F., Angew. Chem. Int. Ed. 2017, 56, 6276-6279.

8. Maiti, D.; Buchwald, S. L., J. Org. Chem. 2010, 75, 1791-1794.

9. Huang, H.; Zhang, G.; Chen, Y., Angew. Chem. Int. Ed. 2015, 54, 7872-7876.

10. Motherwell, W. B.; Vazquez, S., Tetrahedron Lett. 2000, 41, 9667-9671.

11. Shan, G.; Han, X.; Lin, Y.; Yu, S.; Rao, Y., Org. Bio. Chem. 2013, 11, 2318-2322.

12. Rao, M. L. N.; Ramakrishna, B. S., Eur. J. Org. Chem. 2017, 5080-5093.

13. Horne, S.; Rodrigo, R., J. Org. Chem. 1990, 55, 4520-4522.

14. Serratore, N. A.; Anderson, C. B.; Frost, G. B.; Hoang, T.-G.; Underwood, S. J.;

Gemmel, P. M.; Hardy, M. A.; Douglas, C. J., J. Am. Chem. Soc. 2018, 140, 10025-10033.

15. Wang, P.; Rao, H.; Hua, R.; Li, C.-J., Org. Lett. 2012, 14, 902-905.

16. Dreis, A. M.; Douglas, C. J., J. Am. Chem. Soc. 2009, 131, 412-413. 